(C) 2020, American Psychological Association. This paper is not the copy of record and may not exactly replicate the final, authoritative version of the article. Please do not copy or cite without authors' permission. The final article will be available, upon publication, via its DOI: 10.1037/xIm0000848

\title{
Relational rule discovery in complex discrimination learning
}

\author{
Hilary J. Don ${ }^{1,2}$, Micah B. Goldwater ${ }^{1}$, Justine Greenaway ${ }^{1}$, Rosalind Hutchings ${ }^{1}$ \\ \& Evan J. Livesey ${ }^{1}$ \\ ${ }^{1}$ The University of Sydney \\ ${ }^{2}$ Texas A\&M University
}

Please address correspondence to:

Dr Hilary J. Don.

Department of Psychological \& Brain Sciences

Texas A\&M University

4235 TAMU 77843-4235

hilary.don@tamu.edu 


\begin{abstract}
Failure to learn and generalise abstract relational rules has critical implications for education. In this study, we aimed to determine which training conditions facilitate relational transfer in a relatively simple (patterning) discrimination versus a relatively complex (biconditional) discrimination. The amount of training participants received had little influence on rates of relational transfer. Instead, trial-sequencing of the training contingencies influenced relational transfer in different ways depending on the complexity of the discrimination. Clustering instances of relational rules together during training improved transfer of both simpler patterning and more difficult biconditional rules, regardless of individual differences in cognitive reflection. However, blocking all trials of the same type together improved rule transfer only for biconditional discriminations. Individual differences in cognitive reflection were also more predictive of relational rule use under suboptimal training conditions. The results highlight the need for comprehensive accounts of relational learning to consider how learning conditions and individual differences affect the likelihood of engaging in learning relational structures.
\end{abstract}

Keywords: relational transfer; trial-sequencing; complex discriminations; individual differences 
The ability to recognise relational rules and transfer this knowledge to novel contexts is critical for higher-level cognition, and is arguably at the core of what makes humans uniquely intelligent (Halford Wilson, \& Phillips, 2010; Gentner, 2003; Penn Holyoak, \& Povonelli, 2008). However, instead of learning general principles rooted in common relations among stimuli, learners often focus on the features of the particular stimuli encountered during learning and their simpler statistical relations with task-relevant outcomes. This kind of feature-based or associative learning typically generalises to new situations based on surface similarity; experiencing a similar set of stimulus features retrieves associative memories from which predictions about impending events can be made. While feature-based learning and generalization are useful in many contexts (and are abilities shared across the animal kingdom), it appears that learning and transferring relational rules may require a different set of optimal conditions than those that facilitate associative memory.

Recent evidence has shown there is even a trade-off between task conditions that support learning features versus relational rules. For example, when learning novel categories, classification tasks (i.e., when exemplars are presented and the learner needs to guess the category) promote a focus on perceptual features, while inference tasks (i.e., when a partial category exemplar is presented and the learner must infer the missing element) promote a focus on relations among stimulus properties (Goldwater, Don, Krusche \& Livesey, 2018). The current study builds on these findings by examining how people learn novel causal relationships between sets of stimuli (the candidate causes), and their potential effects. In order to succeed in educational settings, students are often required to learn causal relationships of varying complexity, which abide by different scientific and mathematical principles depending on relational aspects of the problem. Many of the major challenges in education can be characterised as understanding how problems are related in terms of their abstract causal principles when their surface similarity is low, while at the same time ignoring 
surface-level similarity between problems that do not share underlying causal principles (Goldwater \& Schalk, 2016). The aim of the current study was to determine how rule-transfer relates to the conditions of learning. Specifically, we examined whether simple manipulations of training parameters facilitate relational transfer, and whether these conditions are equally effective for relational rules of different complexity. Two training conditions that may contribute to rule transfer that are tested here are the amount of training and the sequencing of information provided across training.

\section{Processes of Rule Learning}

Shanks and Darby (1998) developed a task that neatly dissociates generalization on the basis of simple cue-outcome relationships from generalization on the basis of an abstract rule. In their study, participants assumed the role of a doctor whose task was to determine which foods were causing allergic reactions in a fictitious patient. Participants learned the relationships between food cues and the allergic reaction outcome through a process of trial and error. The task design (see Table 1) included examples of a negative patterning discrimination, in which two food cues, presented on separate trials, individually predicted an allergic reaction $(\mathrm{A}+/ \mathrm{B}+)$ but the presentation of those cues in a compound predicted no reaction (AB-), and positive patterning, in which two cues individually predicted no reaction (C-/D-), but in combination predicted an allergic reaction (CD+). Participants can perform accurately on this task by memorizing specific combinations of cues and outcomes. However, the trials conform to an abstract "opposites rule", in which individual cues and their compounds predict opposite results.

In order to assess whether participants learned about the features of the cue-outcome relationships or the relational rule, the training phase also included several incomplete patterning discriminations, comprising either individual cues alone $(\mathrm{I}+/ \mathrm{J}+$ and $\mathrm{M}-/ \mathrm{N}-)$ or compound cues alone (KL- and $\mathrm{OP}+)$. A transfer phase was then administered following 
training, without feedback, which included the remaining items of the incomplete discriminations (e.g. IJ, MN). Taking novel compound IJ as an example, if participants rely on feature-based generalization, they should predict IJ+, due to the surface similarity between IJ and I+/J+. However participants using the opposites rule should predict IJ-, because the components of the compound had individually predicted an outcome. The patterning "opposites" rule is a fairly simple relational rule that many (although not all) participants can readily identify and articulate (Don, Goldwater, Otto \& Livesey, 2015; 2016).

Table 1

Design of patterning task used by Shanks and Darby (1998) and in this paper.

\begin{tabular}{|c|c|c|c|c|}
\hline & \multirow[b]{2}{*}{ Training } & \multirow[b]{2}{*}{ Test } & \multicolumn{2}{|c|}{ Response prediction } \\
\hline & & & Feature & Relation \\
\hline \multirow[t]{4}{*}{ Complete } & $\mathrm{A}+/ \mathrm{B}+/ \mathrm{AB}-$ & $\mathrm{A} / \mathrm{B} / \mathrm{AB}$ & & \\
\hline & C- / D- / CD+ & $\mathrm{C} / \mathrm{D} / \mathrm{CD}$ & & \\
\hline & $\mathrm{E}+/ \mathrm{F}+/ \mathrm{EF}-$ & $\mathrm{E} / \mathrm{F} / \mathrm{EF}$ & & \\
\hline & G- / H- / GH+ & $\mathrm{G} / \mathrm{H} / \mathrm{GH}$ & & \\
\hline \multirow[t]{4}{*}{ Incomplete } & $\mathrm{I}+/ \mathrm{J}+$ & I / J / IJ & $\mathrm{IJ}>\mathrm{MN}$ & $\mathrm{IJ}<\mathrm{MN}$ \\
\hline & KL- & $\mathrm{K} / \mathrm{L} / \mathrm{KL}$ & $\begin{array}{c}\mathrm{O} / \mathrm{P}> \\
\mathrm{K} / \mathrm{L}\end{array}$ & $\begin{array}{c}\mathrm{O} / \mathrm{P}< \\
\mathrm{K} / \mathrm{L}\end{array}$ \\
\hline & $\mathrm{M}-/ \mathrm{N}-$ & $\mathrm{M} / \mathrm{N} / \mathrm{MN}$ & & \\
\hline & $\mathrm{OP}+$ & $\mathrm{O} / \mathrm{P} / \mathrm{OP}$ & & \\
\hline
\end{tabular}

Note: Critical transfer trials are shown in bold. Only the compound transfer trials were included in the calculation of transfer scores in the current study.

Variants of the Shanks-Darby patterning task have been used in several behavioural and neurophysiological studies, with a desire to understand what the dissociation between rule- and feature-based generalization implies for psychological mechanisms (Cobos, Gutiérrez-Cobo, Morís, \& Luque, 2017, Don et al., 2015, 2016; Maes et al., 2015, Milton et al., 2017; Wills et al., 2011a, 2011b). Some of these studies have revealed cognitive resources upon which relational transfer appears to depend (e.g., working memory; Wills et 
al., 2011a, 2011b), and how they build upon lower-level associative mechanisms that remain intact during rule-use (Cobos et al., 2017). While the literature on complex discrimination learning has a tradition of applying formal models of associative learning processes (e.g. Bellingham, Gillette-Bellingham \& Kehoe, 1985; Thorwart, Livesey \& Harris, 2012; Wagner \& Brandon, 2001; Whitlow \& Wagner, 1972) and typically recognises that distinct processes underlie relational rule-learning, little attempt has been made so far to apply a formal computational framework to capture these rule learning processes ${ }^{1}$. Thus another goal of the current study is to move towards an account of the cognitive processes underlying the discovery and application of the relational rules.

Theories of relational learning and transfer from the literature on analogical reasoning provide one approach to explaining relational rule transfer in complex discriminations (e.g., see Doumas, Hummel, \& Sandhofer, 2008; Falkenheiner, Forbus, \& Gentner, 1989; Kokinov \& Petrov, 2001; Holyoak \& Thagard, 1989, for several computational approaches). The general account from this literature is that the explicit analogical comparison of pairs of example problems, passages of text, or clusters of visual stimuli, involves an alignment of the constitutive elements based on the correspondences within their respective relational structures. In the case of the patterning discrimination (with $\mathrm{A}+, \mathrm{B}+, \mathrm{AB}-, \mathrm{C}-, \mathrm{D}-, \mathrm{CD}+$ ), that would involve aligning $\mathrm{A}+$ and $\mathrm{B}+$ with $\mathrm{C}$ - and $\mathrm{D}-$, and then $\mathrm{AB}$ - with $\mathrm{CD}+$, respectively.

The literature on analogical learning shows that after structural alignment, the common relational structure is abstracted and ready to be transferred to novel contexts (e.g., Gick \& Holyoak, 1983; Cantrabone \& Holyoak, 1989; Goldwater \& Gentner, 2015). For example, in Gentner, Loewenstein and Thompson (2003), one group of business students aligned a pair of cases concerning conflicts between business parties that were resolved with

\footnotetext{
${ }^{1}$ One exception to this observation is Verguts and Fias (2009), who developed a Serial Recurrent Network that appears to have the capacity to learn feature- and rule-based transfer using the same complex learning operations.
} 
the same negotiation strategy. A second group of business students read the same pair of cases but did not explicitly align them. Then all the students were presented a novel unresolved conflict and the task was to propose a solution. The students who aligned the prior cases were more likely to spontaneously apply the strategy utilised in them than the students who just read the cases sequentially.

In the case of the patterning task, the incomplete discriminations serve as the novel context to which the relational structure should be readily applicable; mapping this structure onto the contingencies that have been learned (i.e. either the individual cue or compound trials) provides the means to make predictions about the novel trial types on the basis of the relational rule rather than simple featural similarity.

\section{Effects of training}

Shanks and Darby (1998) found that participants who learned patterning discriminations slowly during training were more likely to rate transfer items based on featural similarity, whereas participants who learned the discriminations quickly during training were more likely to show rule-based generalization. One potential explanation for this result is that noticing the patterning rule may facilitate accurate responding during training, such that participants who notice the rule may perform more accurately than those who do not. Shanks and Darby instead argued that the effect of training accuracy on rule transfer demonstrates that rule-use relies on the formation of the basic associations upon which the rule applies. Thus, with sufficient training, all participants should be capable of learning the rule. If this is the case, then a greater length of training may increase the opportunity to learn the associations, and therefore provide a greater probability of extracting the rule. Nevertheless, Shanks and Darby suggested that rule complexity and individual differences may also affect rule learning.

\section{Effects of trial-sequencing}


More recently, processes of comparison and structural alignment have been examined in category learning tasks, which are supervised learning tasks with a similar structure to complex discrimination learning. Most category learning tasks present sequences of exemplars that alternate between categories unsystematically, for instance by randomizing in blocks. This means that, for a task involving two categories, exemplars from one category are approximately equally likely to be followed by an exemplar of the same category versus an exemplar of the alternate category. However, to specifically understand the role of comparison, experimenters have intentionally manipulated exemplar sequences with the assumption that learners compare stimuli and feedback across consecutive trials.

Interleaving (or intermixing) sequences, when exemplars are more likely to alternate between categories on consecutive trials, is suggested to lead to better learning because it supports between category discrimination, highlighting differences between exemplars from different categories (Bjork et al., 2013). Like other causal learning procedures, most versions of the Shanks-Darby task randomly intermix all training trial types together in blocks. When the goal is to maximise learning of (and memory for) simple cue-outcome relationships, this procedure is well justified. Intermixing trial types ensures that they are relatively well distributed, and spaced learning trials generally produces better retention than massed learning trials (Ebbinghaus, 1885).

However, there are some indications that blocking stimulus presentation, that is, presenting sequences of consecutive exemplars from the same category, is better for certain types of category structures and learning conditions. Specifically, blocking exemplars appears to be beneficial when within-category exemplar similarity is low, as it allows withincategory comparisons on successive trials (e.g. Carvalho \& Goldstone, 2014; 2015).

Within-category similarity is often low when the category is defined by a relational rule, as opposed to shared perceptual features, because the perceptual features are a more 
salient driver of similarity than the more abstract relational rule (see Gentner \& Kurtz, 2005). Goldwater et al. (2018) have recently shown that the sequence of presentation of exemplars influences whether participants are more likely to generalise based on relational rules, or on perceptual features in a category learning task. In Experiment 2 of this study, category exemplars were presented in sequences that were either more blocked (low probability of alternating between categories) or more intermixed (high probability of alternating between categories). They found that blocking exemplars facilitated rule-based responding on ambiguous transfer trials, whereas intermixing trials resulted in more feature-based responses. One interpretation of this result is that the blocked sequences facilitated a structural alignment of exemplars on consecutive trials, supporting relational rule discovery. There is a growing interest in how trial sequences affect learning and generalization, for both the development of learning theories, but also by making clear connections to educational practice (Carvalho \& Goldstone, 2014; Rohrer \& Pashler, 2010; Goldwater \& Schalk, 2016). However, this research typically does not consider how the complexity of what is learned may affect optimal sequences.

\section{Rule complexity}

To assess how training conditions influence relational transfer of rules of different complexity, we compared performance in the patterning task with performance in a different complex discrimination that is not linearly solvable. In a biconditional discrimination, four cues are presented as four pairwise compounds. Two of these compounds predict the outcome (e.g., $\mathrm{AB}+, \mathrm{CD}+$ ), and two do not (AC-, BD-), such that no individual cue is uniquely predictive of an outcome (Saavedra, 1975). Both human participants and laboratory animals find learning biconditional discriminations relatively difficult; they are acquired more slowly than linearly solvable discriminations that are matched for presentation of compound cues (Livesey, Thorwart, de Fina, \& Harris, 2011; Saavedra, 1975) but also more 
slowly than positive and negative patterning discriminations (Harris \& Livesey, 2008; Harris, Livesey, Ghareai, \& Westbrook, 2008).

Baetu, Burns, Yu \& Baker (2018) recently argued that biconditional discriminations cannot be solved using a rule. However, there is a relational structure within a biconditional discrimination that can be abstracted and applied to novel stimuli. This relational structure is more complex than the relations present in patterning and representing it in abstract form is arguably much more difficult than is the case with the opposites rule. Harris \& Livesey (2008) compared patterning and biconditional tasks, introducing biconditional transfer trials (e.g. QR-O1, QS-O2, ST?). They found that fewer participants gave relational responses to biconditional transfer trials than patterning transfer trials, and that fewer participants reported awareness of the biconditional rule than the patterning rule. Nevertheless, a minority of participants did report awareness of the biconditional rule and/or rated the biconditional transfer trials according to the rule. Thus, although biconditional discriminations appear to be more difficult to learn and transfer, it is possible for participants to learn the relational structure and generalise this structure to new stimuli.

Livesey, Don, Uengoer and Thorwart (2019) found evidence that participants acquire knowledge about the relational structure of the discrimination in which a cue appears (e.g. linearly solvable uniconditional vs biconditional) and transfer that knowledge to new learning situations that involve the same cue. This study provides some evidence of learning and generalising relational qualities of the biconditional discrimination, though this learning was still generalised on the basis of the surface features (i.e. the cues) involved in those discriminations in the first place.

Using patterning and biconditional discriminations therefore provides a point of comparison for the effects of training conditions on relational rules of different complexity. 
Table 2

Design of biconditional task

\begin{tabular}{|c|c|c|c|c|}
\hline \multicolumn{5}{|c|}{ Biconditional Discrimination } \\
\hline & \multirow[b]{2}{*}{ Training } & \multirow[b]{2}{*}{ Test } & \multicolumn{2}{|c|}{ Response prediction } \\
\hline & & & Feature & Relation \\
\hline \multirow[t]{4}{*}{ Complete } & $\mathrm{AB}+/ \mathrm{BC}-/ \mathrm{CD}+/ \mathrm{AD}-$ & $\mathrm{AB} / \mathrm{BC} / \mathrm{CD} / \mathrm{DA}$ & & \\
\hline & EF-/ FG+ / GH- / HE+ & $\mathrm{EF} / \mathrm{FG} / \mathrm{GH} / \mathrm{HE}$ & & \\
\hline & IJ+ / JK- / KL+ / LI- & IJ / JK / KL / LI & & \\
\hline & $\mathrm{MN}-/$ NO+ / OP- / PM+ & $\mathrm{MN} / \mathrm{NO} / \mathrm{OP} / \mathrm{PM}$ & & \\
\hline \multirow[t]{2}{*}{ Incomplete } & $\mathrm{QR}+/ \mathrm{RS}-/ \mathrm{ST}+$ & $\mathrm{QR} / \mathrm{RS} / \mathrm{ST} / \mathrm{QT}$ & $\mathrm{QT}>\mathrm{UX}$ & QT $<$ UX \\
\hline & UV- / VW+ / WX- & $\mathrm{UV} / \mathrm{VW} / \mathrm{WX} / \mathbf{U X}$ & & \\
\hline
\end{tabular}

\section{The current study}

This study aimed to test the training conditions that affect relational learning and transfer. In all experiments, participants either received training in patterning discriminations (Table 1) or biconditional discriminations (Table 2). To test transfer of the biconditional rule, we designed a biconditional task similar to the Shanks \& Darby task. This biconditional task included four complete discriminations (e.g. AB+/BC-/CD+/AD-) as well as two incomplete discriminations (e.g., QR+/RS-/ST+). The test phase also included the trained cues and the remaining items from the incomplete discriminations (e.g. TQ). Thus, we can tease apart responding based on summation of features (TQ+) and on the relational rule (TQ-) in a similar way to patterning.

A summary of the experimental manipulations is shown in Table 3. In experiment 1 , we tested whether greater amounts of training increases rule use in both patterning and biconditional discrimination tasks. As researchers have argued that all participants should be capable of learning the relational rule, given sufficient training of the cue-outcome associations (Shanks \& Darby, 1998), we predicted that greater amounts of training should result in increased rule transfer. 
Table 3

Summary of experimental manipulations

\begin{tabular}{lllll}
\hline Exp & Discrimination & Manipulation & Training & Covariate \\
\hline 1A & Patterning & Training length & Fixed & Questionnaire \\
1B & Biconditional & Training length & Fixed & Questionnaire \\
2A & Patterning & Trial sequence & Fixed & CRT \\
2B & Patterning & Trial sequence & Trials-to-criterion & CRT, Ravens \\
3A & Biconditional & Trial sequence & Fixed & CRT \\
3B & Biconditional & Trial sequence & Trials-to-criterion & CRT, Ravens \\
\hline
\end{tabular}

Given that trial sequencing appears to affect relational discovery and transfer in other learning domains, we also investigated whether particular sequences of trials are effective in enhancing rule transfer in complex discriminations, comparing simpler (patterning) and more difficult (biconditional) relational rules. Experiment 2 focused on relational transfer in patterning discriminations, after fixed length training (Experiment $2 \mathrm{~A}$ ) or trained to a performance criterion (Experiment 2B), while Experiment 3 focused on relational transfer in biconditional discriminations, again after fixed-length (Experiment 3A) and trials-to-criterion (Experiment 3B). Although the comparison process is different in the case of complex discriminations than comparisons between exemplars of categories - the predicted outcome depends on interrelationships between trial types that comprise a discrimination set, not interrelationships between features of the exemplar itself - fostering comparison with the discrimination set may be important for learning and abstracting relational information about the structure of a complex discrimination. Intermixing trials generally produces better memory retention and discrimination between trials. However, according to structural alignment models, intermixing may prevent relational rule discovery. Structural alignment is a process that depends on working memory (e.g., see Tohill \& Holyoak, 2000), and temporally separating elements of a complete discrimination set may prevent learners from noticing the relational rule, and instead rely on associative processes to memorise the cueoutcome combinations. Therefore, a blocked sequence in which all elements of complete 
discrimination sets are presented consecutively and repeatedly may actually maximise relational rule discovery.

Although blocking trials within a discrimination set should allow the relevant structural relations to be identified more readily, repeating those blocks does not afford the alignment of distinct sets that share the same relational structure, as this requires comparison across multiple examples of the same relational structures. To more precisely track the predictions of a structural alignment account, we developed a third, novel "clustered" condition. In this case, the blocked sequencing applied to a grouping of each trial type within a discrimination (creating a cluster to highlight the relational rule), but then each discrimination set as a whole was intermixed with clusters of the other discrimination sets. Structural alignment predicts this should maximise learning because it both supports actively considering the whole discrimination set in working memory with consecutive trials, but then further shows how the rule applies to multiple discrimination sets, supporting alignment and abstraction across them.

The three types of trial sequencing that we used are illustrated schematically in Figure 1 and described in more detail below. These manipulations applied only to the complete discriminations. Incomplete discriminations were always presented in random order following training of complete compounds.

Intermixed. In the intermixed condition, all elements from each of the complete discriminations were presented in a random order within each block (see Figure 1A). Each element was presented twice per block.

Blocked. In the blocked condition, all presentations of the elements from only one complete discrimination were presented in one block (see Figure 1B). For example, all 12 repetitions of $A+/ B+/ A B-$ were presented within a block, randomised in subsets of trials containing all trial types within a given discrimination. For example, one repetition may have 


\section{a) Intermixed}

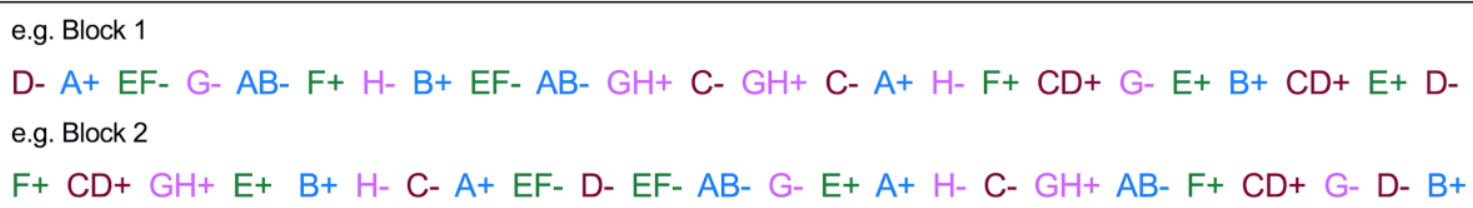

\section{b) Blocked}

e.g. Block 1

$A+A B-B+B+A+A B-A+A+B+A B-B+A B-A B-B+A+A B-B+A+A+A B-A B-B+B+A+\ldots$ e.g. Block 2

D- C- CD+ D- D- C- D- CD+ D- C- C- CD+ C- CD+ C- C- D- CD+ CD+ D- D- C- CD+ C- ..

\section{c) Clustered}

e.g. Block 1

$B+A+A B-G-G H+H-D-C D+C-E+F+E F-G H+H-G-F+E F-E+C D+C-D-A+B+A B-$ e.g. Block 2

D- C- CD+ F+EF- E+ A+ AB- B+ G- H- GH+AB-A+B+EF- F+ E+C-CD+D- GH+ H- G-

Figure 1. Schematic of block structure in each of the trial-sequencing conditions. Letters denote different cues or trials, colours denote different discriminations. In a) the intermixed condition, each trial type from each discrimination is presented in random order within a block. In b) the blocked condition, all presentations of the trial types from one discrimination were presented within one block. In c) the clustered condition, all trial types from each discrimination were presented within a block, but the trials were grouped according to the discrimination they belonged to.

the sequence, $\mathrm{A}+, \mathrm{AB}-, \mathrm{B}+$, and then the next may have $\mathrm{AB}-, \mathrm{B}+, \mathrm{A}+$. This was followed by all 12 presentations of C-/D-/CD+, with blocks alternating between positive and negative patterning examples.

Clustered. In the clustered condition, all elements from each of the discriminations were presented within a block, but trials were sequenced in 'clusters' of elements from the same discrimination (see Figure 1C). For example, a sequence of trials may be $\mathrm{AB}-, \mathrm{A}+, \mathrm{B}+$ D-, CD+, C-.

The experiments were designed to inform the development of a theory than can integrate associative and relational learning processes in the context of complex discrimination learning. In general, a structural alignment account predicts that the clustered condition should produce the best relational rule learning. However, it is silent about how the 
benefits of this sequence may further depend on the quantity of training or complexity of the relational rule to be learned.

Individual Differences. There is increasing evidence of stable individual differences in the propensity to use rule-based transfer across learning tasks, (e.g., McDaniel, Cahill, Robbins, \& Wiener, 2014; Little \& McDaniel, 2015; Don et al., 2016). These individual differences have been shown to interact with task structure in their effects on learning features versus relational rules (Goldwater et al., 2018). There is similar evidence that individual differences in cognitive capacity are related to rule learning in complex discrimination tasks. For example, Maes, Vanderoost, D’Hooge, De Houwer \& Beckers (2017) found that participants with higher Ravens Progressive Matrices (RPM) scores, a test of general fluid intelligence, were more likely to show rule-based generalization in a patterning task. Similarly, Wills et al. (2011b) found that participants who scored higher on a measure of complex working memory (OSPAN; Turner \& Engle, 1989) were more likely to display rule transfer in the patterning task. Baetu et al. (2018) measured participants' performance on a wider range of fluid abilities and also gave them either patterning or biconditional discriminations to solve sequentially. They found that a number of fluid abilities (most consistently, reasoning ability as measured by RPM and the Comprehensive Abilities Battery - Induction, Hakstian \& Cattell, 1975) were related to both rule transfer in the patterning task and performance on the second of two complex discriminations that shared the same relational structure as the first. Further, performance on the Cognitive Reflection Test (CRT; Frederick, 2005), predicts rule-based learning in the patterning task (Don et al., 2016). The CRT measures an individual's tendency to inhibit immediately available intuitive responses and engage in deliberative processes, and has been shown to be a significant predictor of performance on heuristic-and-biases tasks (Toplak, West \& Stanovich, 2011). The CRT includes three mathematical problems that trigger an intuitive 
incorrect answer, for example, "A bat and a ball cost $\$ 1.10$ in total. The bat costs a dollar more than the ball. How much does the ball cost?" Answering this question correctly requires the participant to engage in cognitive reflection in order to override the intuitive incorrect response of "10 cents". The correct solution, "5 cents" is mathematically simple to derive once the intuitive response is rejected. The CRT is thought to gauge critical thinking and is thus related to both thinking style and cognitive capacity (and does correlate with measures of general intelligence such as RPM, e.g. Gray \& Holyoak, 2019). Therefore, in the current work, we included both RPM (Experiments $2 \mathrm{~b}$ and $3 \mathrm{~b}$ ) and the CRT to measure individual differences in cognition.

In Experiment 1, we assessed how well participants could verbalise the patterning or biconditional rules, using a short questionnaire including both open response and forced choice components. We expect there to be a relationship between the ability to accurately report the relevant rule and showing transfer based on that rule. Nevertheless, prior research has shown that this may be a necessary, but not sufficient, condition for rule transfer (Don et al., 2016).

\section{Experiment 1}

Experiment 1 aimed to compare rates of rule transfer as a function of training length for both the standard patterning discrimination, and the new biconditional discrimination. It also aimed to assess whether participants show relational rule transfer in a biconditional discrimination. Participants received 6,10 or 14 repetitions of each trial type in training comprising either patterning discriminations (Experiment 1A) or biconditional discriminations (Experiment 1B), all in intermixed trial sequencing. We predicted that more participants would show rule-based generalization with greater amounts of training, as once the associations are well learned, there is greater opportunity to abstract the relational rule. 
To assess awareness of the relational rules, we included a post-experimental questionnaire similar to that used by Harris \& Livesey (2008).

\section{Method}

\section{Participants}

144 undergraduate students (90 female, mean age $=20.79, S D=4.50)$ from the University of Sydney participated in return for partial course credit, or monetary compensation. Approval for the study was obtained from the University of Sydney Human Research Ethics Committee. Participants were randomly allocated to each training length group in Experiment 1A and 1B $(n=24)$.

\section{Apparatus \& Stimuli}

The experiment was programmed using PsychToolbox for Matlab (Kleiner, Brainard, \& Pelli, 2007), and was run on Apple Mac Mini computers. Cue stimuli included 300 x 300 pixel images of coffee, banana, fish, lemon, cheese, garlic, apple, eggs, peanuts, mushrooms, strawberry, milk, bread, avocado, broccoli, olive oil, cherries, butter, chocolate, carrots, peach, bacon, peas and prawns with accompanying labels in blue text beneath each image. Foods were randomly allocated to cues for each participant. All images were presented on the upper half of the screen. Compound cues were horizontally aligned and the configuration of compound cues on screen (left vs. right) was counterbalanced within each block. The allergic reaction and no allergic reaction outcome options were presented in boxes aligned vertically on the lower half of the screen.

\section{Procedure}

Training. Participants were asked to assume the role of a doctor whose task was to determine which foods were causing allergic reactions in a fictitious patient, Mr X. On each trial, one or two food cues were presented and participants were required to predict whether an allergic reaction would occur by choosing from "no allergic reaction" and "ALLERGIC 
REACTION" outcome options. When an outcome was selected, the options disappeared, and corrective feedback was provided while the food cues remained on the screen. The correct answer appeared, accompanied by either the word "CORRECT" in green, or "INCORRECT" in red, depending on the accuracy of the prediction. Participants were instructed that at first they would have to guess, but that using the feedback provided, their accuracy should improve over time. There were either three, five, or seven blocks of training, according to training length group, with two repetitions of each trial type per block.

Test. Following training, participants were instructed to use the knowledge they had gained so far in order to rate how likely it was that an allergic reaction would occur, given their patient had eaten the presented foods. On each trial, one of the test items (see Table 1 and 2) was presented, and participants were asked to rate the likelihood that an allergic reaction would occur on a 10-point linear analogue scale ranging from "definitely WILL NOT occur" to "definitely WILL occur". Although most previous studies on patterning have used binary responses in the transfer phase, we have previously found this scale to be successful in measuring transfer in the patterning task (Don et al., 2016), and it allows us to assess rule use on a more continuous scale. Participants were able to adjust their rating before pressing the spacebar to continue (without feedback). Critically, the test phase included transfer trials that did not appear during training. These were the remaining items from the incomplete discriminations. In the patterning group, the six transfer trials were $\mathrm{IJ}, \mathrm{MN}, \mathrm{K} / \mathrm{L}$ and O/P. In the biconditional condition the transfer trials were QT and UX. For ease of comparison between discrimination conditions, analysis of patterning transfer included only the compound trials IJ and MN. Two blocks of the test phase were completed, with each test item presented once per block.

Manipulation check. After completing the test phase, participants were given a paperbased questionnaire to assess their knowledge of the patterning or biconditional rule. The first 
component asked participants to verbalise any rule they noticed during the task. The second required participants to give a yes/no response to two questions based on those used by Harris \& Livesey (2008) to assess whether participants had noticed the relational rules. Participants learning the patterning discrimination (Experiment 1A) were asked:

Did you notice that if A predicted an allergic reaction, and B predicted an allergic reaction, then the combination of $A$ and $B$ predicted no allergic reaction? (negative patterning)

and,

Did you notice that if A predicted no allergic reaction, and B predicted no allergic reaction, then the combination of $A$ and $B$ predicted an allergic reaction? (positive patterning).

As participants in Harris \& Livesey (2008) were less likely to report awareness of a biconditional rule than a patterning rule, we used two different descriptions of the biconditional rule in order to potentially capture different understanding of the relational structure. These were:

Did you notice that if $A$ and $B$ together resulted in an allergic reaction, and $C$ and $D$ together resulted in an allergic reaction, then $A$ and $C$ or $B$ and $D$ together resulted in no allergic reaction?

And,

Did you notice that if Food A resulted in an allergic reaction when eaten with food B, then it would result in no reaction when eaten with food D? Similarly, iffood C resulted in no reaction when eaten with food $B$, then it would result in an allergic reaction when eaten with food $D$ ? 


\section{Results}

As the training and transfer trials differed between Experiment $1 \mathrm{~A}$ and 1B, we cannot compare performance directly. However in order to be more comparable, we assessed only the compound transfer trials for patterning (IJ and MN). Analysis of the single cue transfer trials and combined transfer scores are included in the Supplemental material.

\section{Experiment 1A: Patterning}

Training. Accuracy during training and mean transfer score for each group is shown in Figure 2. There was a significant increase in accuracy across training in the 6-rep, $F(2,46)$ $=81.63, p<.001, \eta_{p}^{2}=.78,10-$ rep $, F(4,92)=78.77, \mathrm{p}<.001, \eta_{p}^{2}=.774$, and 14-rep, $F(6,138)$ $=94.23 \mathrm{p}<.001, \eta_{p}^{2}=.804$, training groups. We also compared accuracy in the final block of training between groups. Although there was an increase in accuracy in the expected direction given the extent of training, with mean accuracy at $.84, .90$, and .92 for the 6-rep, 10-rep, and 14-rep groups, respectively, this difference did not reach significance, $F(2,69)=$ $2.76, p=.07, \eta_{p}^{2}=.074$

Transfer. Transfer scores were calculated from the difference in mean ratings for $\mathrm{MN}$ and IJ transfer trials (MN-IJ), where higher positive scores indicate greater rule-transfer. 44\% of participants showed a positive transfer score. There was no significant effect of training group on transfer score, $F<1$. We ran a Bayesian ANOVA in JASP with default priors to determine whether there was evidence for the null effect of training length on transfer scores. This revealed substantial evidence for the null, $\mathrm{BF}_{01}=6.26$. In addition to testing the effect of training length on the degree of rule transfer, we also tested whether more training increased the number of participants displaying rule transfer. The proportion of participants who showed a positive transfer score were 10/24, 10/24 and 12/24 for the 6-rep, 10-rep and 14-rep groups, respectively. A Pearson Chi-Square Test showed that there was no association between training group and a positive transfer score, $X(2)=450, \mathrm{p}=.799$. 

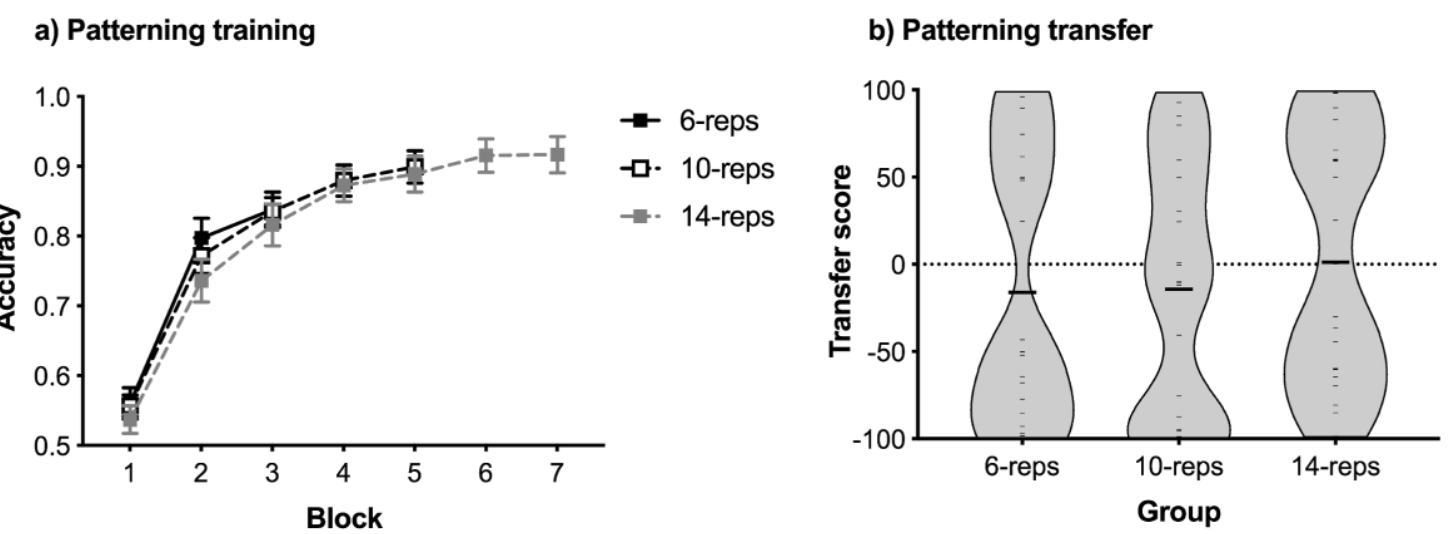

Figure 2. Results from a) training and b) transfer for the patterning discrimination (Experiment 1A). Error bars in panel A represent standard error (SE). The bean plots in Panel $\mathrm{B}$ show mean and individual transfer scores calculated over the novel trial types shown during transfer test. Positive scores indicate rule-consistent predictions, whereas negative scores indicate feature-consistent predictions.

Trained discriminations. Learning scores for the trained elements from complete and incomplete compounds are shown in Figure 3. These scores were calculated by taking the average ratings for the trials paired with an allergic reaction minus the average for the trials paired with no reaction, and reflect how well participants recall the outcome paired with the trained cues. There was no significant effect of group on learning scores for either the complete, $F(2,69)=2.93, p=.06, \eta_{p}^{2}=.078$, or incomplete, $F(2,69)=2.22, p=.116, \eta_{p}^{2}=$ .06 , trained items. Table 4 shows correlations between transfer score, training accuracy, and performance on the complete and incomplete discriminations at test. There was a significant correlation between transfer and performance on the complete discriminations at test only, $r=$ $.387, \mathrm{p}<.001$. See Supplementary Material for further analysis of the learning scores. 


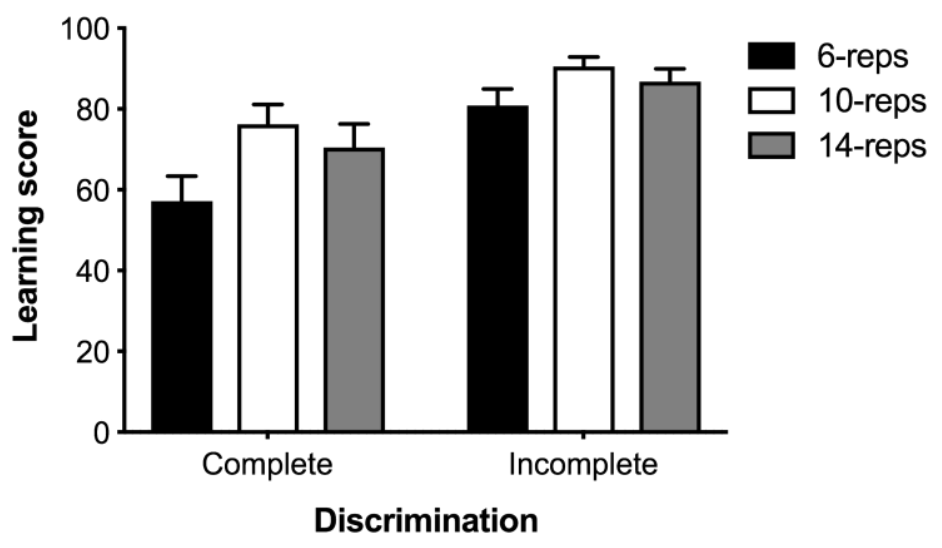

Figure 3. Learning scores for the trained items from the complete and incomplete discriminations from Experiment 1A. Scores were calculated from the difference between ratings for cues paired with the outcome and ratings for cues that were not paired with the outcome.

Table 4.

Correlation matrix for all patterning participants in Experiment 1A

\begin{tabular}{|c|c|c|c|c|c|}
\hline & & Transfer & $\begin{array}{l}\text { Training } \\
\text { accuracy }\end{array}$ & $\begin{array}{c}\text { Test } \\
\text { complete }\end{array}$ & $\begin{array}{c}\text { Test } \\
\text { incomplete }\end{array}$ \\
\hline \multirow[t]{3}{*}{ Training accuracy } & $r$ & .191 & & & \\
\hline & $p$ & .109 & & & \\
\hline & $N$ & 72 & & & \\
\hline \multirow[t]{3}{*}{ Test complete } & $r$ & .387 & .709 & & \\
\hline & $p$ & $<.001$ & $<.001$ & & \\
\hline & $N$ & 72 & 72 & & \\
\hline \multirow[t]{3}{*}{ Test incomplete } & $r$ & -.143 & .337 & .438 & \\
\hline & $p$ & .232 & .004 & $<.001$ & \\
\hline & $N$ & 72 & 72 & 72 & \\
\hline
\end{tabular}

Post-experimental questionnaire. In the open response component of the postexperimental questionnaire, participants received 2 points for reporting either an opposites rule, e.g. that foods presented in compound caused the opposite reaction of those foods presented individually or vice versa, or both the positive and negative patterning rules separately, e.g. two non-allergenic foods combined caused an allergic reaction, and two allergenic foods combined caused no reaction (43 participants). Participants received 1 point for reporting either the positive or negative patterning rule, but not both (10 participants), and the remaining participants were given a score of 0 (19 participants). The number and mean 
transfer scores for participants who did and did not report at least one patterning rule (score > 0) are shown in Table 5. Performance in the open response component of the questionnaire was significantly correlated with rule transfer, $r=.525, p<.001$, such that participants who could accurately verbalise the patterning rule were more likely to apply that rule. However, consistent with Don et al. (2016), the ability to report the patterning rule appears to be necessary but not sufficient for rule generalization. Of the 53 participants who accurately reported at least one rule, 22 showed a negative transfer score, indicating transfer based on features, rather than the rule. Of the 19 participants who did not report either rule, only one showed positive rule transfer. Although the ability to report the patterning rule increased with additional training (see Supplementary Material), there was no significant association between training group and reporting the patterning rule, $X(4)=5.58, \mathrm{p}=.233$.

In the forced choice component of the questionnaire, 59 participants reported that they had noticed both patterning rules (score $=2$ ), and a further 12 participants noticed at least one of the positive or negative patterning rules (score $=1$ ). Only one participant reported that they did not notice either rule (score $=0$ ). Performance on the forced response component was not correlated with transfer scores, $r=.108, p=.368$, and did not differ according to training group, $X(4)=3.64, p=.458$.

Table 5 .

Number of participants showing rule transfer (positive transfer score) and feature transfer (negative transfer score), and their mean transfer scores, based on ability to verbalise at least one patterning rule in the open response component of the questionnaire. The total row refers to the overall transfer score for participants within each column

\begin{tabular}{lccccccc}
\hline & \multicolumn{3}{c}{+ Report } & & \multicolumn{3}{c}{ - Report } \\
\cline { 2 - 4 } \cline { 6 - 8 } & $\mathrm{N}$ & Mean & SEM & & $\mathrm{N}$ & Mean & SEM \\
\hline Rule transfer & 31 & 65.70 & 6.62 & & 1 & 24.37 & - \\
Feature transfer & 22 & -60.87 & 5.17 & & 18 & -78.81 & 6.39 \\
Total & 53 & 13.16 & 9.55 & & 19 & -73.38 & 8.12 \\
\hline
\end{tabular}

\section{Experiment 1B: Biconditional}


Training. Accuracy during training and mean transfer score for each group in the biconditional discrimination is shown in Figure 4. There was a significant increase in accuracy across training in the 6-rep, $F(2,46)=29.27, p<001 ., \eta_{p}^{2}=.56,10$-rep, $\mathrm{F}(4,92)$ $=48.32, p<.001, \eta_{p}^{2}=.678$, and 14-rep, $F(6,138)=40.51, p<.001, \eta_{p}^{2}=.638$, training groups. In this case, accuracy in the final block of training significantly differed between groups, $F(2,69)=5.93, p=.004, \eta_{p}^{2}=.147$. Accuracy in the 10-rep group $(\mathrm{M}=.76)$ was significantly greater than the 6-rep group $(\mathrm{M}=.64), \mathrm{F}(1,46)=8.26, \mathrm{p}=.006, \eta_{p}^{2}=.152$, but there was no difference in final block accuracy between the 10-rep group and the 14-rep $\operatorname{group}(\mathrm{M}=.79), F<1$.

Transfer. Transfer scores were calculated from the difference between mean ratings for XU and TQ transfer trials (XU-TQ, see Table 2), where higher positive scores indicate greater rule-transfer. Thirty percent of participants showed a positive transfer score, but there was no significant effect of group on transfer score, $F<1$. A Bayesian ANOVA revealed substantial evidence for a null effect of training group, $\mathrm{BF}_{01}=5.13$. The proportion of positive transfer scores were 5/24, 7/24 and 10/24 for the 6-rep, 10-rep and 14-rep groups, respectively. A Pearson Chi-Square Test showed that there was no association between training group and a positive transfer score, $X(2)=2.49, \mathrm{p}=.288$.

a) Biconditional training

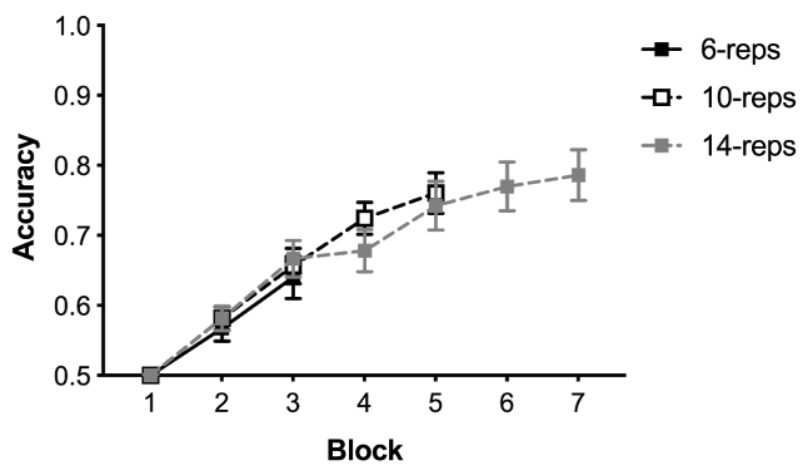

b) Biconditional transfer

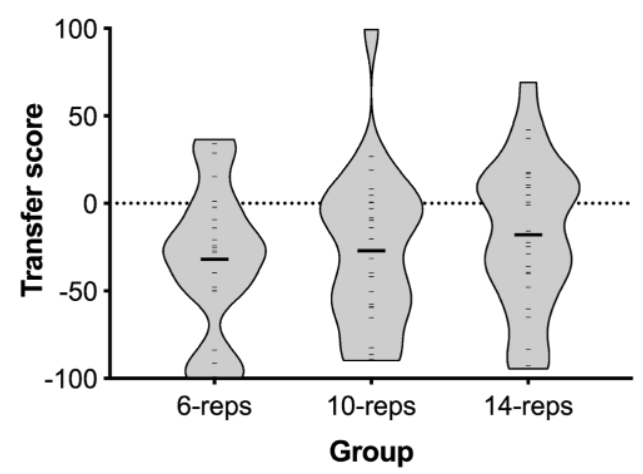

Figure 4. Results from a) training and b) transfer for the biconditional discrimination (Experiment 1A). Error bars in panel A represent standard error of the mean. Solid bars in panel B represent mean transfer scores for each group. 
Trained discriminations. Learning scores for complete and incomplete trained items are shown in Figure 5. In this case, there was a significant effect of group in both complete, $F(2,69)=5.13, p=.008, \eta_{p}^{2}=.129$, and incomplete, $F(2,69)=6.79, p=.002, \eta_{p}^{2}=.164$, discriminations. Analysis of simple effects showed that the 10-rep group showed higher learning scores than the 6-rep group for both complete, $t(46)=2.33, p=.024$, and incomplete, $t(46)=3.153, p=.003$, discriminations, but there was no difference between the 10-rep and 14-rep groups in either measure, highest $t(46)=.98, p=.333$. Table 6 shows correlations between transfer score, training accuracy, and performance on the complete and incomplete discriminations at test. There were no significant correlations between transfer score and any measure of training performance.

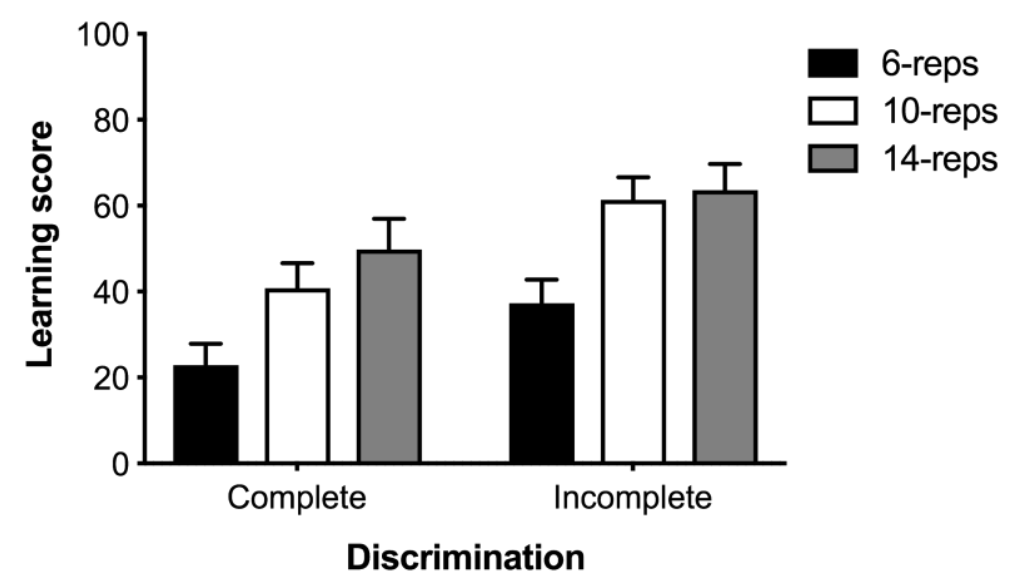

Figure 5. Learning scores at test for the trained items from the complete and incomplete discriminations from Experiment 1B. 
Table 6.

Correlation matrix for all biconditional participants in Experiment 1B

\begin{tabular}{llcccc}
\hline & & Transfer & Training & Test & Test \\
accuracy & complete & incomplete \\
\hline Training accuracy & $r$ & .063 & & & \\
& $p$ & .598 & & & \\
& $N$ & 72 & & & \\
\hline Test complete & $r$ & -.003 & .758 & & \\
& $p$ & .979 & $<.001$ & & \\
& $N$ & 72 & 72 & & \\
Test incomplete & $r$ & .177 & .816 & .786 & \\
& $p$ & .137 & $<.001$ & $<.001$ & \\
& $N$ & 72 & 72 & 72 & \\
\hline
\end{tabular}

Post-experimental questionnaire. Responses to the open-response component of the post-experimental questionnaire were scored based on increasing approximations of the biconditional rule. Questionnaire data was missing for one participant. Participants received 2 points for reporting the full biconditional rule (10 participants), for example, that individual foods were both allergenic and non-allergenic, depending on their combination with other foods. One point was received for reporting that the combination of foods was important, without specifying that individual foods related both to the presence and absence of an allergic reaction conditionalized on other foods. (10 participants). Participants received 0.5 points for reporting specific examples that indicated an understanding of the relevance of cue combinations, and were consistent with the biconditional rule e.g. "bread + egg $=$ allergic reaction, bread + milk $=$ no reaction, milk + peach $=$ allergic reaction" $(4$ participants $)$. All other responses received a score of 0 (47 participants). In this case, the correlation between transfer score and responses in the open component of the post-experimental questionnaire did not quite reach significance, $r=.231, p=.053$. Table 7 shows the number of participants showing positive and negative transfer scores, and their mean transfer scores, based on whether participants reported a biconditional rule (or any approximation thereof) in the open component of the questionnaire. There was no association between training group and 
reporting the biconditional rule, $X(6)=4.22, \mathrm{p}=.647$. The ability to report some approximation of the biconditional rule was also not sufficient for rule transfer. Of the 24 participants with a positive report score, 14 did not show relational transfer.

In the forced-choice component of the questionnaire, forty-six participants reported noticing at least one of the descriptions of the biconditional rule. Neither description of the biconditional rule was more likely to be recognised than the other, with 32 yes responses to each description, of which 18 participants responded yes to both descriptions. There was no correlation between responses to either of the forced choice questions and rule transfer, highest $r=.193, p=.107$. As each of the response options described the biconditional rule in a different way, and reporting that they noticed both does not necessarily indicate greater knowledge of the rule, we gave participants a score of 1 if they noticed at least one of the biconditional rules, and 0 otherwise. Performance on this component of the manipulation check was also not significantly associated with training group $X(2)=3.53, \mathrm{p}=.172$.

Table 7.

Number of participants showing rule transfer (positive transfer score) and feature transfer (negative transfer score), and their mean transfer scores, based on ability to report some element of the biconditional rule (a positive score) in the open component of the questionnaire. The total row refers to the overall transfer score for participants within each column

\begin{tabular}{lccccccc}
\hline & \multicolumn{3}{c}{+ Report } & & \multicolumn{3}{c}{ - Report } \\
\cline { 2 - 4 } \cline { 7 - 8 } & $\mathrm{N}$ & Mean & SEM & & $\mathrm{N}$ & Mean & SEM \\
\hline Rule transfer & 10 & 31.89 & 9.90 & & 12 & 14.89 & 3.80 \\
Feature transfer & 14 & -40.15 & 7.99 & & 35 & -49.96 & 5.12 \\
Total & 24 & -10.14 & 9.58 & & 47 & -33.40 & 5.72 \\
\hline
\end{tabular}

\section{Discussion}

In Experiment 1, participants received either 6, 10 or 14 repetitions of the critical training trials. The amount of training had no strong effect on the rate of rule transfer based on the relational structures present in either a patterning or a biconditional task. This is somewhat surprising, as Shanks \& Darby (1998) suggest that all participants should be 
capable of learning the patterning rule given sufficient training. For the patterning discrimination, there were no significant group differences in participants' memory of the trained contingencies during the test phase. For the biconditional discrimination, the two longer training groups showed improved recall of the trained contingencies, however, this did not result in improved relational transfer. This result suggests the amount of exposure to complex discriminations does not strongly impact the use of rule transfer at test, at least within the ranges of training length that we examined in this study.

We also found poorer training performance and rates of relational transfer for a biconditional rule compared to a patterning rule, which is consistent with the results found by Harris \& Livesey (2008). Participants were also less likely to report awareness of the biconditional rule, or to accurately verbalise the biconditional rule, compared to the patterning rule. Despite the lower rate of rule transfer of the biconditional discrimination, it is possible to learn and transfer the relations that comprise this complex task. Around a third of participants completing the biconditional discrimination gave higher ratings to XU than TQ, demonstrating transfer based on relational structure. Replicating the finding from Don et al (2016), the ability to verbalise the patterning rule appears to be necessary, but not sufficient for rule transfer. This relationship was not found so clearly for the biconditional rule, where around half the participants with a positive transfer score could not verbalise the rule. Nevertheless, of the participants who showed positive rule transfer, those who could verbalise the biconditional rule showed higher transfer scores than those who could not (see Table 7). The ability to report the patterning and biconditional rules were not improved by a greater amount of training.

Perhaps in order to see a strong effect of the amount of training, we would need to have greatly increased the number of training repetitions in the longest condition, especially for the biconditional discrimination. In any case, within the range of training lengths over 
which participants perform relatively well and clearly show evidence of learning, training length has little effect on rule transfer. Note, however, that our results are not necessarily in conflict with Shanks and Darby (1998). They showed an association between learning efficiency and rule transfer which could indicate a direct causal relationship (e.g., strong learning results in rule transfer), or an indirect relationship (e.g. rule discovery results in both high performance and rule transfer). Our results suggest that the opportunity the participant is given to learn the discrimination is not a strong determinant of rule transfer, which does not fit well with the direct causal hypothesis. This observation informed the design of Experiments 2 and 3, in which we employed a fixed training length for half the participants and trials-to-criterion training for half of the participants, to ensure good acquisition of each of the complete discrimination sets.

\section{Experiment 2}

In Experiment 2, participants learned complete patterning discriminations in either intermixed, blocked or clustered conditions. Experiment 2A had a fixed training length, with all participants completing 12 repetitions of training of the complete discriminations. ${ }^{2}$

Following this training, all participants received 12 repetitions of the incomplete discriminations, where all trials were presented in an intermixed fashion. Experiment $2 \mathrm{~B}$ trained participants to a performance criterion (described below). This training was followed by a fixed 12 repetitions of incomplete discriminations, which were again presented in an intermixed fashion, regardless of group. All participants therefore had matched exposure to the trial types on which the transfer trials were based. Their training history differed only on the contingencies from which relational rules could be learned and abstracted.

\footnotetext{
2 We chose 12 repetitions based on the results of Experiment 1, since there were no significant differences in memory for the trained contingencies between 10 and 14 repetitions of training for either patterning or biconditional discriminations, but 10 repetitions led to better memory than 6 repetitions for the biconditional discrimination.
} 
We expected improvements in rule transfer in the blocked and clustered conditions compared to the intermixed condition. Goldwater et al. (2018) found that blocking trials of exemplars from the same category increased rates of relational transfer compared to intermixed presentation of the same exemplars, as this may allow greater comparison within instances of the relational rule. Clustering trials may also facilitate this form of comparison, but has the additional benefit of providing more opportunities to make comparisons across different instances of the relation, which we hypothesised may make other critical processes in structural alignment easier for the learner to perform. We therefore predicted that both forms of manipulated trial sequencing (i.e. blocked and clustered) would allow for greater rule transfer than standard intermixed sequencing but that the clustered condition may produce more rule transfer than the blocked condition. Analyses therefore focused on these planned comparisons.

\section{Method}

\section{Participants}

We aimed to recruit 40 usable participants in each trial sequencing group of Experiments $2 \mathrm{~A}$ and $2 \mathrm{~B}$. This sample size was based on that used in previous studies to assess trial spacing (Goldwater et al., 2018, Experiment 2) and patterning (Don et al., 2015). Two-hundred and forty-one undergraduates from the University of Sydney (169 female, mean age $=20.8, S D=5.1$ ) participated in return for partial course credit. Of these participants, 120 received fixed training (Experiment 2A), and 121 received trials-to-criterion training (Experiment 2B). One participant did not reach the learning criterion after 12 blocks (24 repetitions) of the patterning discriminations and was removed from all analyses. The study was approved by the University of Sydney Human Research Ethics Committee.

\section{Apparatus and Stimuli}

Apparatus and Stimuli were identical to Experiment 1. 


\section{Procedure}

Experiment 2 used the same allergist task as in Experiment 1, and responding to training and test trials occurred in the same manner. The same complete and incomplete patterning discriminations were also used, but the order of presentation of the complete discriminations differed between groups. In the intermixed group, all trials from each of the complete discriminations were presented twice within a block, in random order. In the blocked condition, all presentations of elements from one discrimination were presented within a block, followed by all presentations of elements from another discrimination, with the constraint that the discriminations alternated from one block to the next between negative patterning and positive patterning. In the clustered group, all elements from each discrimination were presented twice within a block (as in the intermixed condition), but the trial types were clustered so that each of the three different elements from one discrimination (e.g. $\mathrm{A}+, \mathrm{B}+$ and $\mathrm{AB}-$ ) were presented consecutively. Within each of these clusters of trials, the elements could be presented in any order (e.g., A+, AB-, then $\mathrm{B}+$ ), and within a block, clusters from each of the four discriminations were presented twice (e.g., two clusters of cues A and B, two clusters of cues C and D, etc.) The order of presentation of each of these clusters was also randomised (e.g., cues $\mathrm{G}$ and $\mathrm{H}$ first; cues $\mathrm{A}$ and $\mathrm{B}$ next, etc.) Two clusters of the same elements were allowed to follow consecutively, though a switch to a cluster with different elements was much more likely.

In Experiment 2A, participants received a fixed length of training, with 12 presentations of each of the components from each complete discrimination. For instance, in the blocked condition, all 12 presentations of each element of one discrimination were presented. In Experiment 2B, participants received a minimum of three blocks of training of each discrimination, following which participants were trained to a criterion of 7/8 correct responses, applied to each complete discrimination separately. For instance, once participants 
reached 7/8 correct for $\mathrm{A}, \mathrm{B}$ and $\mathrm{AB}$ trials, these trials were no longer presented during training, and participants were trained on the remaining discriminations until they reached criterion, or a maximum of 12 blocks. To match criteria between the patterning group (three components per discrimination) and biconditional group (four components per discrimination) groups, the compounds from each complete patterning discrimination were presented twice, which is not uncommon in work on patterning discriminations in human and animal learning (e.g., Harris et al., 2008; Harris \& Livesey, 2008). Incomplete discriminations were presented following training of the complete discriminations. All groups received 12 repetitions of all trial types that were presented intermixed, regardless of trialsequencing group. The test phase was identical to Experiment 1, and was identical for each group, with all trials presented in intermixed random order.

Analysis. To assess our hypotheses for the effects of trial-sequencing, we ran planned contrasts for both training and transfer phases. These contrasts compared the intermixed group to the average of the blocked and clustered groups, and the blocked group to the clustered group. The remaining pairwise comparisons of the transfer scores are presented in the Supplementary Material.

\section{Results}

\section{Fixed training (Experiment 2A)}

Complete discriminations. Accuracy for complete discriminations across training is shown in Figure 6A. For comparison between conditions, performance is graphed according to the number of presentations of each trial type. The intermixed and clustered conditions contained two presentations of each trial type per block, and so to make the visual presentation of the blocked condition comparable, we present the average of two presentations of each trial type at each data point in the figure and for the analyses. Because 
all presentations of the same trial type occurred within one block in the blocked group, these analyses will better reflect performance across each individual block, rather than across the training phase as a whole. That is, in the blocked condition accuracy increased within a block, but then regressed on the next block because a new set of items was introduced. In the intermixed and clustered conditions, the increase in accuracy rates over the course of the learning phase was monotonic. These figures make the three conditions directly comparable by focusing on learning about individual items.

3 x 6 mixed measures ANOVA was run with group (Intermixed vs. Blocked vs. Clustered) as a between subjects factor, and repetition in bins of 2 presentations of each trial type (1-6) as a within subjects factor. There was a significant linear effect of bin, indicating an increase in accuracy over presentations, $F(1,117)=751.01, p<.001, \eta_{p}^{2}=.865$. There was also a significant main effect of group, $F(2,117)=32.01, p<.001, \eta_{p}^{2}=.354$, and a significant interaction between bin and group, $F(10,585)=13.96, p<.001, \eta_{p}^{2}=.193$. Contrast analyses showed that accuracy was poorer overall for the intermixed group compared to the average of blocked and clustered groups, $F(1,117)=30.96, p<.001, \eta_{p}^{2}=$ .209. The blocked group performed significantly better than the clustered group, $F(1,117)=$ $33.05, p<.001, \eta_{p}^{2}=.22$.

Incomplete discriminations. Training accuracy for incomplete discriminations is shown in Figure 6B. There was a significant linear effect of block $F(1,117)=686.19, p<$ $.001, \eta_{p}^{2}=.854$, and an interaction between block and group, $F(2,117)=8.75, p<.001, \eta_{p}^{2}=$ .13. There was also a significant main effect of group, $F(2,117)=4.62, p=.012, \eta_{p}^{2}=.073$. Contrast analyses showed that there was no significant difference in accuracy between the intermixed and the average of blocked and clustered groups, $F<1$, but the clustered group had significantly higher accuracy than the blocked group, $F(1,117)=8.59, p=.004, \eta_{p}^{2}=$ .068 . 
a) Complete discriminations: fixed

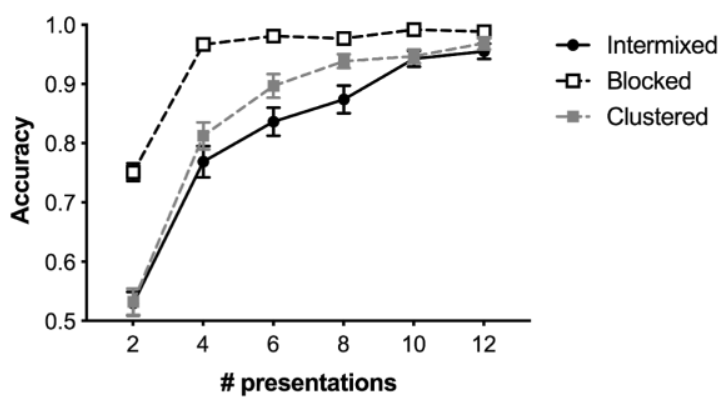

c) Complete discriminations: criterion

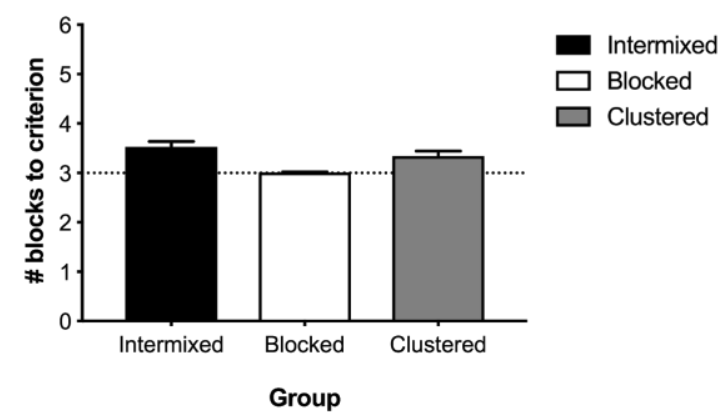

b) Incomplete discriminations: fixed

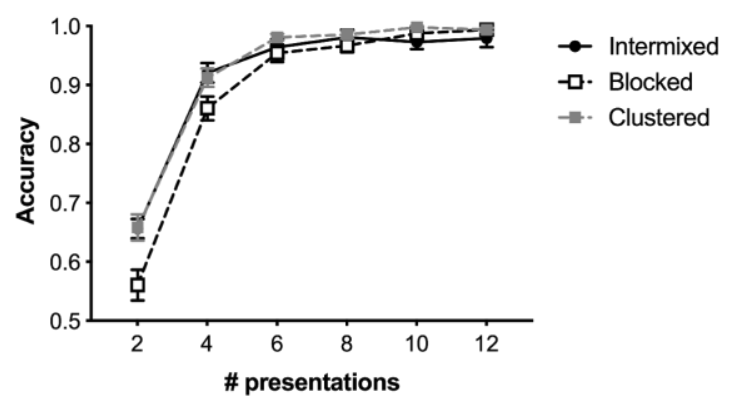

d) Incomplete discriminations: criterion

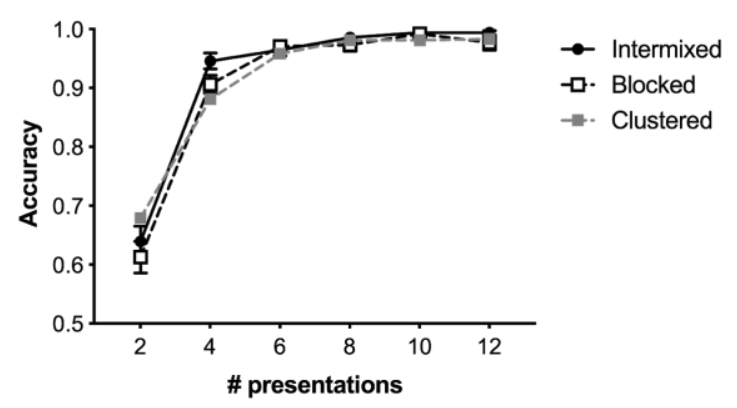

Figure 6. Patterning training performance for complete (left panels) and incomplete (right panels) for fixed training (top panels) and trials-to-criterion training (bottom panels).

\section{Trials to criterion training (Experiment 2B)}

Complete discriminations. The mean number of blocks to reach criterion for complete discriminations is shown in Figure 6C. A one-way ANOVA with group as a between subjects factor showed a significant main effect of group, $F(2,117)=8.83, p<.001$, $\eta_{p}^{2}=.734$. Contrast analyses showed that the intermixed group required significantly more blocks of training to reach criterion than the average of the blocked and clustered groups, $F(1,117)=10.49, p=.002, \eta_{p}^{2}=.082$. The blocked group required significantly fewer blocks to reach criterion than the clustered group, as all but one subject reached criterion within the minimum 3 blocks, $F(1,117)=7.17, p=.008, \eta_{p}^{2}=.058$.

Incomplete discriminations. Training accuracy for incomplete discriminations is shown in Figure 6D. A $6 \times 3$ ANOVA revealed a linear effect of presentation bin $F(1,117)=$ 
$686.19, p<.001, \eta_{p}^{2}=.854$. There was no significant main effect or interaction with group, $F s<1$.

\section{Transfer test}

Transfer scores overall and for each training condition are shown in Figure 7. We ran a 3 x 2 between subjects ANOVA with group (interleaved vs. blocked vs. clustered) and training method (fixed training vs. trials-to-criterion) as factors. This revealed a significant main effect of group $F(2,234)=3.72, p=.026, \eta_{p}^{2}=.031$. There was no significant effect of training method, $F(1,234)=.271, p=.603, \eta_{p}^{2}=.001$, and no interaction between group and training method, $F(2,234)=.071, p=.931, \eta_{p}^{2}=.001$. Contrasts for group were therefore run collapsed across the fixed and trials-to-criterion conditions. There was no significant difference in transfer scores between the intermixed group and the average of blocked and clustered groups, $F(1,234)=.787, p=.376, \eta_{p}^{2}=.003$, however the clustered group had significantly higher transfer scores than the blocked group, $F(1,234)=6.65, p=.011, \eta_{p}^{2}=$ .028. While the clustered group had the greatest number of participants showing rule transfer, a chi-square test showed that there was no significant association between trialsequencing group and the number of participants who showed rule transfer, $X(2)=2.54, p=$ .281 . 
a) Patterning overall

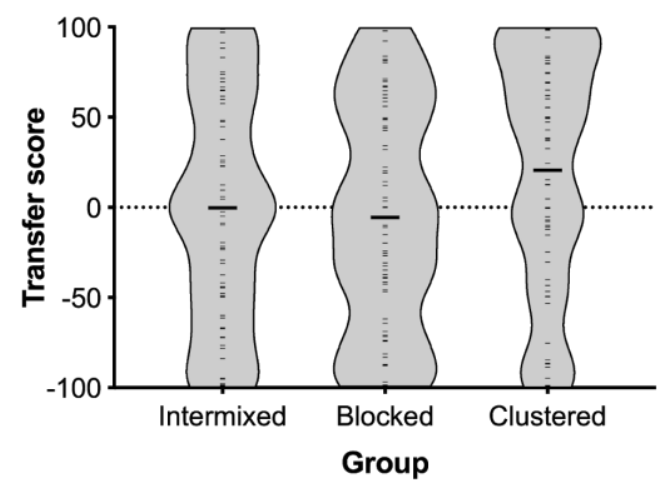

b) Patterning fixed

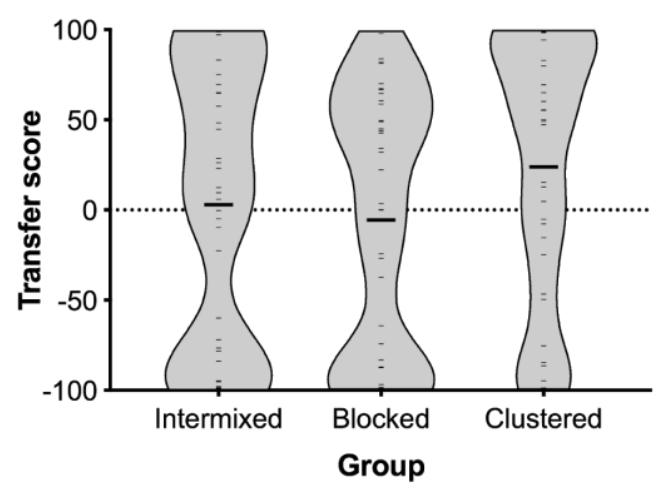

c) Patterning criterion

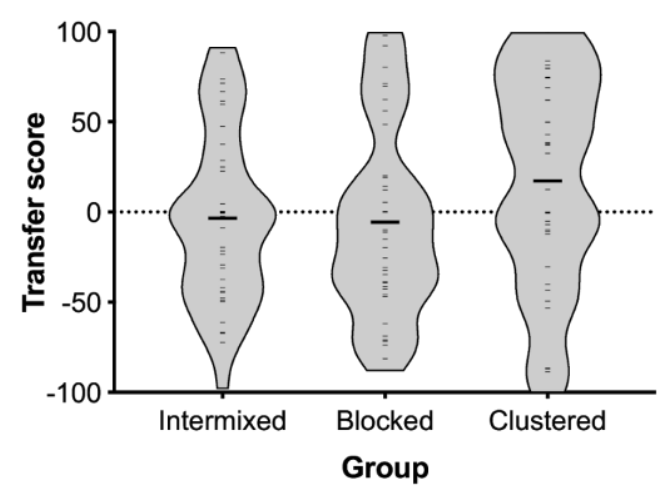

Figure 7. Transfer scores for A) all patterning participants, B) fixed training participants, and C) trials-to-criterion training. Solid bars represent the mean transfer scores for each group.

To determine whether the trial-sequencing conditions affect memory for learned associations, we also compared learning scores for the trained items from the complete and incomplete discriminations at test (see Figure 8). For the complete discriminations, learning scores were significantly better in the intermixed condition than the average of the two experimental conditions, $F(1,234)=37.33, p<.001, \eta_{p}^{2}=.138$, however this was largely due 
to there being substantially worse test performance in the blocked condition, which displayed significantly poorer scores than the clustered condition, $F(1,234)=72.89, p<.001, \eta_{p}^{2}=$ .237. Neither contrast was significant for the trained items from the incomplete discriminations, highest $F(1,234)=2.74, p=.099, \eta_{p}^{2}=.012$.
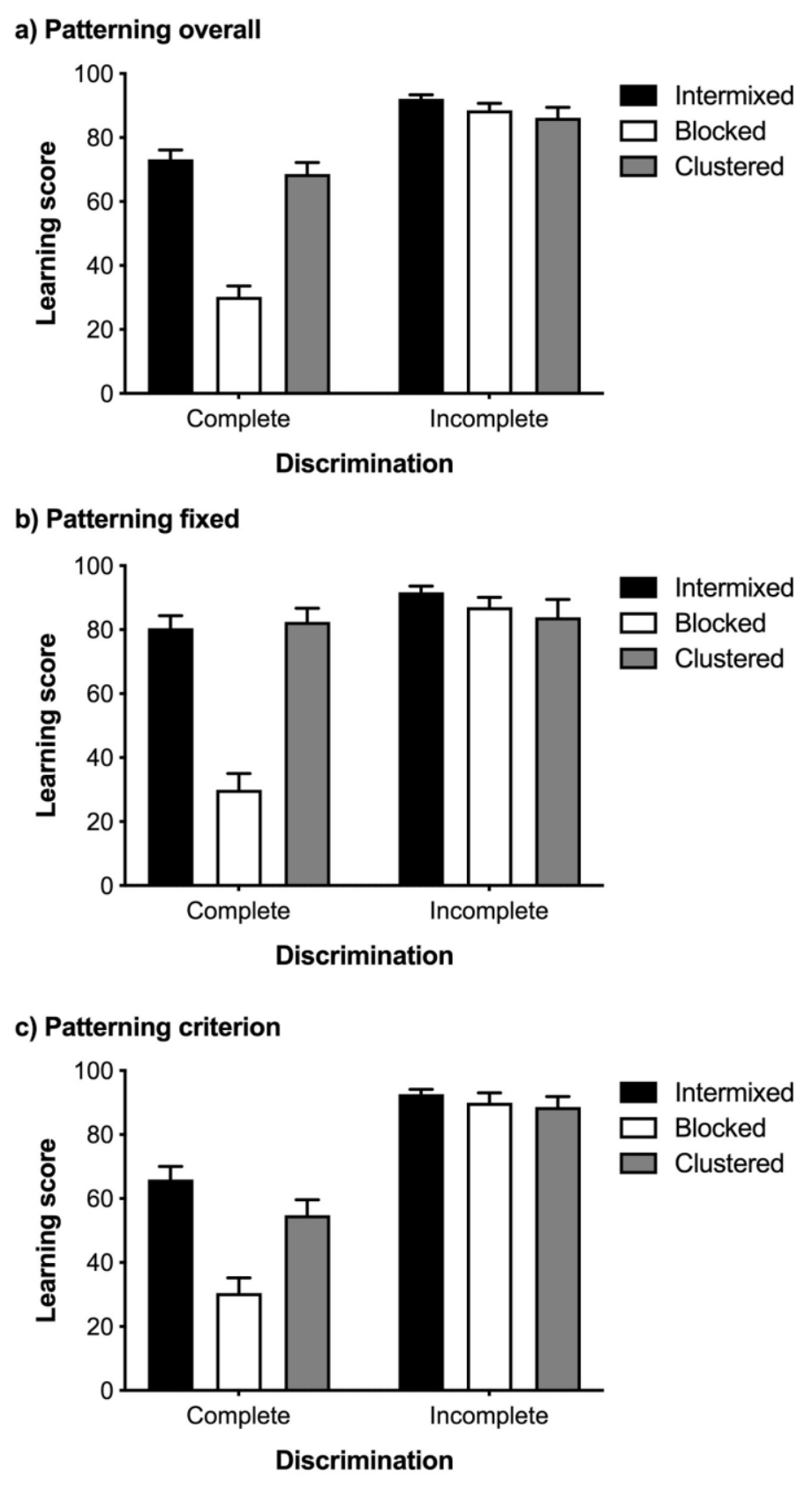

Figure 8. Learning scores at test for the trained items from the complete and incomplete patterning discriminations a) overall, b) in the fixed training condition, and c) in the trials-tocriterion training criterion. 


\section{Individual differences}

Correlations between relational transfer scores and individual difference measures, as well as performance on other items in the test phase, are shown in Table 8. Collapsed across all participants, there was a significant correlation between the number of correct CRT responses and rule transfer, $r=.153, p=.018$. Broken down by group, the correlation between CRT and transfer was driven primarily by the intermixed group, $r=.359, p=.001$, with no significant correlation observed in the blocked group, $r=.075, p=.508$, or clustered group, $r=.11, p=.329$.

There was no significant correlation with RPM, $r=.153, p=.096$, however it is worth noting that RPM was only collected for Experiment $2 b$, and therefore has less power. Correlations for the individual training groups ranged from $r=.075, p=.508$ for the blocked group up to $r=.216, p=.180$ for the intermixed group. Transfer score did correlate with performance on test items from complete discriminations.

Table 8.

Correlation matrix for all patterning participants

\begin{tabular}{ccccccc}
\hline & & Transfer & CRT & Ravens & $\begin{array}{c}\text { Test } \\
\text { complete }\end{array}$ & $\begin{array}{c}\text { Test } \\
\text { incomplete }\end{array}$ \\
\hline CRT & $r$ & $\mathbf{. 1 5 3}$ & - & & & \\
& $p$ & .018 & & & & \\
& $n$ & 240 & & & & \\
\hline Ravens & $r$ & .153 & $\mathbf{. 3 8 5}$ & - & & \\
& $p$ & .096 & $<.001$ & & & \\
& $n$ & 119 & 119 & & & \\
Test complete & $r$ & $\mathbf{. 3 3 5}$ & $\mathbf{. 1 7 9}$ & $\mathbf{. 1 9 1}$ & - & \\
& $p$ & $<.001$ & .005 & .037 & & \\
\hline Test incomplete & $r$ & 240 & 240 & 119 & & \\
& $r$ & -.002 & $\mathbf{. 1 6 4}$ & .037 & $\mathbf{. 2 8 4}$ & - \\
& $p$ & .981 & .011 & .693 & $<.001$ & \\
& $n$ & 240 & 240 & 119 & 240 & \\
\hline
\end{tabular}

\section{Discussion}

The results from Experiment 2 indicate that trial-sequencing has an effect on ruletransfer in patterning. Interestingly, despite much better performance during training, we did 
not see any evidence that the blocked condition produced more relational transfer than a standard intermixed version of the patterning task. Instead, this group showed the weakest rule transfer after both fixed length training and training to criterion, and significantly weaker rule transfer than the clustered condition. In contrast, clustering trials led to the greatest amount of rule transfer, which is in line with our hypotheses (see Supplementary Material for pairwise comparisons). There was no significant association between group and the number of participants who showed positive transfer scores. Instead, trial-sequencing appeared to affect the strength of rule-transfer scores, rather than increasing the number of rule-users.

We also found that memory for the complete training items at test was significantly correlated with rule transfer, such that those who could more accurately recall the outcome paired with the trained items from the complete discriminations were more likely to rate the remaining items from the incomplete discriminations according to the patterning rule. This is perhaps unsurprising, as knowledge of the rule would both help performance during training and on the test trials. The blocked group showed the poorest memory for the trained complete discriminations, which could provide an explanation for the poor rule-transfer in this group. We will discuss this issue in further detail in the general discussion.

Participants with greater cognitive reflection were also more likely to show rule-based transfer. This relationship was most evident in the standard intermixed condition, where the correlation $(r=.359)$ was comparable to our previous findings. Don et al. (2016), for instance, found a positive correlation $(r=.427)$ between CRT and relational transfer in a similar (intermixed trial) patterning task. Interestingly, there was no significant relationship between rule transfer and performance on RPM. Maes et al. (2017) reported a modest positive relationship $(r=.25)$ between Ravens performance and a similar version of patterning rule transfer. Our results are not strongly inconsistent with this observation as we too found weak positive correlations $(r=.153$ overall, and $r=.216$ for the intermixed 
version) but these did not reach statistical significance. On the whole, it seems likely that the relationship between Ravens and rule transfer in the patterning task is weakly positive.

\section{Experiment 3}

Experiment 1 showed that relational transfer of biconditional discriminations is possible, but more difficult than for patterning discriminations. We therefore wanted to test whether the same trial-sequencing manipulations have the same effect on relational transfer in a biconditional discrimination. Experiment 3A and 3B were identical in most respects to Experiment $2 \mathrm{~A}$ and $2 \mathrm{~B}$, respectively. The only difference was that participants were trained on biconditional discriminations instead of patterning discriminations.

In addition to the potential for lower rates of relational transfer in Experiment 3 than in Experiment 2, there is another potential difference to consider. In Experiment 2, the blocked sequence produced high accuracy during the learning phase, and then low memory accuracy and rule transfer during test. One potential explanation for this discrepancy in performance is that within each block, the task could be solved with a simple shortcut that relies on the number of cues present on a given trial to predict the outcome. For example, a participant may learn that for block $x$, any trial with a single cue predicts an allergic reaction whereas a compound trial predicts no allergic reaction. This shortcut effectively eliminates the need to commit to memory which cue leads to which outcome. For a biconditional discrimination however, this number-of-cues shortcut cannot be used to solve the task, and so the blocked condition may show a distinct pattern of results in Experiment 3 compared to Experiment 2.

\section{Participants}

\section{Method}


A further 249 undergraduates from the University of Sydney (170 female, mean age $=$ $20.1, S D=3.3)$ participated in return for partial course credit, again with the aim of having 40 usable participants in each trial spacing group of Experiments $3 \mathrm{~A}$ and $3 \mathrm{~B}$. Of these participants, 120 received fixed training (Experiment 3A), and 129 received trials-to-criterion training (Experiment 3B). Nine participants in Experiment 3B did not reach the learning criterion after 12 blocks ( 24 repetitions) of the biconditional discrimination and were excluded from analyses. The study was approved by the University of Sydney Human Research Ethics Committee.

\section{Apparatus \& Stimuli}

Apparatus and stimuli were identical to previous experiments.

\section{Procedure}

The procedure was very similar to Experiment 2, but used the cue-outcome contingencies shown in Table 2. Participants were trained on complete biconditional discriminations in intermixed, blocked, or clustered conditions, followed by 12 repetitions of the incomplete discriminations in intermixed random order. Participants in Experiment 3A received 12 repetitions of fixed training of the complete discriminations, and Experiment 3B were trained to criterion in the same manner as Experiment 2B. All participants received the same test phase where test trials were presented intermixed and in random order.

\section{Results}

\section{Fixed training (Experiment 3A)}

Complete discriminations. Accuracy for complete biconditional discriminations across training is shown in Figure 9A. A 3 x 6 ANOVA mixed measures ANOVA with group and repetition bin as factors revealed a linear effect of repetition bin, $F(1,117)=362.90, p<$ $.001, \eta_{p}^{2}=.756$, but no interaction with group, $F(2,117)=2.18, p=.117, \eta_{p}^{2}=.036$. There 
was a significant main effect of group, $F(2,117)=44.28, p<.001, \eta_{p}^{2}=.431$. The intermixed group again performed significantly poorer overall than the average of the blocked and clustered groups, $F(1,117)=57.66, p<.001$, and the blocked group performed significantly better than the clustered group, $F(1,117)=30.90, p<.001, \eta_{p}^{2}=.209$.

Incomplete discriminations. Accuracy for incomplete biconditional discriminations across training is shown in Figure 9B. A 3 x 6 ANOVA revealed a significant linear effect of repetition bin, $F(1,117)=276.74, p<.001, \eta_{p}^{2}=.703$, a significant main effect of group, $F(2,117)=4.51, p=.013, \eta_{p}^{2}=.071$, but no significant interaction between repetition and group, $F(2,117)=2.95, p=.056, \eta_{p}^{2}=.048$. Contrasts showed no significant difference between the intermixed group and the average of the blocked and clustered groups, $F(1,117)$ $=1.89, \mathrm{p}=.172, \eta_{p}^{2}=.016$, however accuracy was significantly better for the blocked than the clustered group, $F(1,117)=7.12, \mathrm{p}=.009, \eta_{p}^{2}=.057$.

a) Complete discriminations: fixed

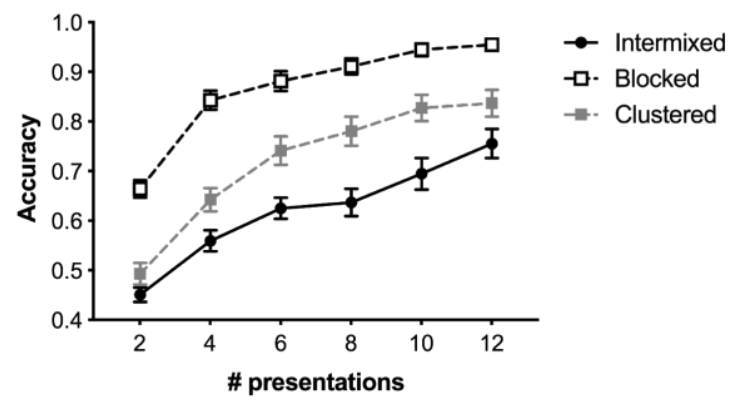

c) Complete discriminations: criterion

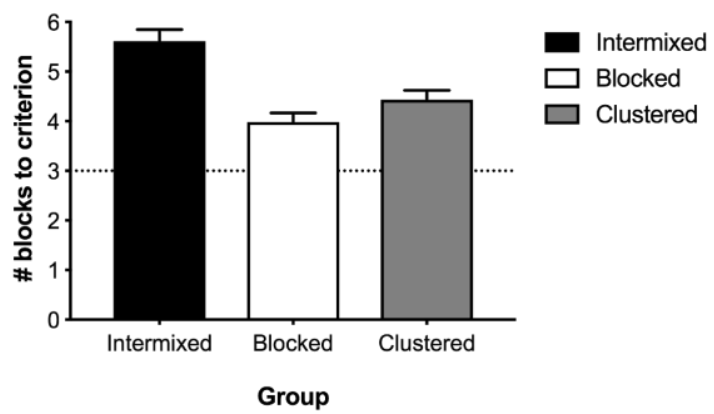

b) Incomplete discriminations: fixed

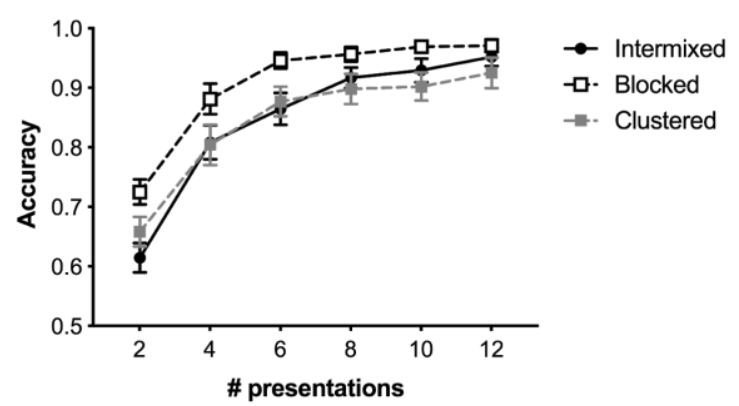

d) Incomplete discriminations: criterion

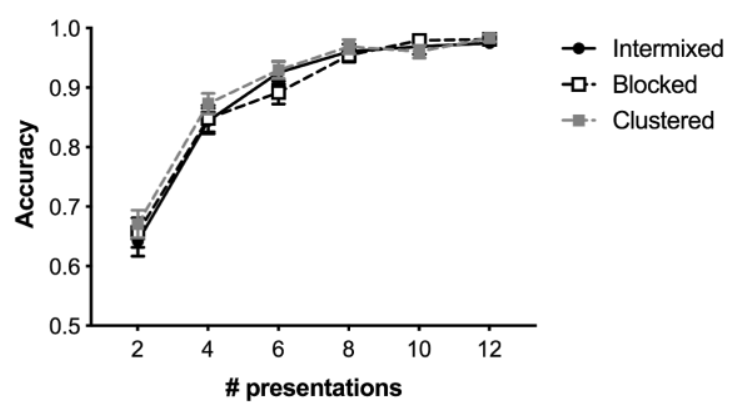

Figure 9. Biconditional training performance for complete (left panels) and incomplete (right panels) for fixed training (top panels) and trials-to-criterion training (bottom panels). 


\section{Trials-to-criterion (Experiment 3B)}

Complete discriminations. The mean number of blocks to reach criterion for complete discriminations is shown in Figure 9C. A one-way ANOVA with group as a factor revealed a significant main effect of group, $F(2,117)=16.82, p<.001, \eta_{p}^{2}=.223$. The intermixed group required significantly more blocks of training to reach criterion than the blocked and clustered groups, $F(1,117)=31.23, p<.001, \eta_{p}^{2}=.211$, but there was no significant difference between blocked and clustered groups, $F(1,117)=2.40, p=.124, \eta_{p}^{2}=$ .02 .

Incomplete discriminations. Accuracy across training for incomplete compounds is shown in Figure 9D. A 3 x 6 mixed measures ANOVA revealed a significant linear effect of repetition bin, $F(1,117)=480.67, p<.001, \eta_{p}^{2}=.804$, but there was no significant main effect of group, or interaction between repetition bin and group, $F s<1$. 
a) Biconditional overall

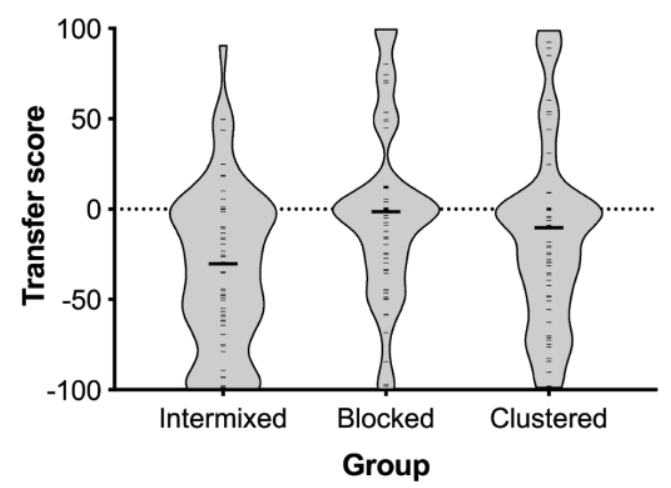

b) Biconditional fixed

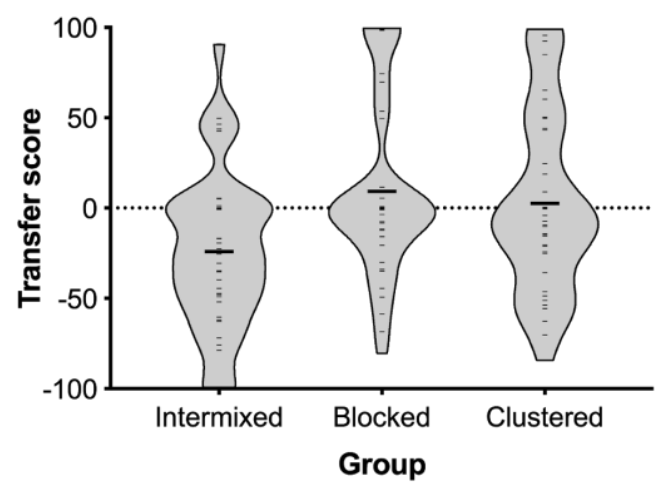

c) Biconditional criterion

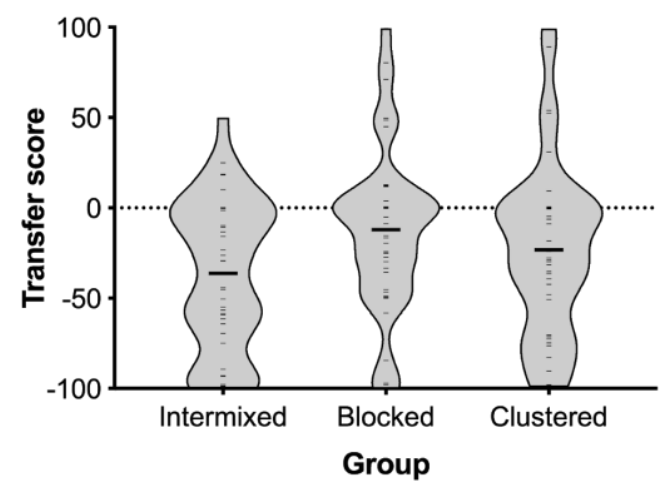

Figure 10. Transfer scores for A) all biconditional participants, B) fixed training participants, and $\mathrm{C}$ ) trials-to-criterion training.

\section{Transfer test}

Transfer scores overall and for each training condition are shown in Figure 10. A $3 \mathrm{x}$ 2 between subjects ANOVA with group (interleaved vs. blocked vs. clustered) and training (fixed training vs. trials-to-criterion) as factors revealed a significant main effect of group, $F(2,234)=8.12, p<.001, \eta_{p}^{2}=.065$. In this case, there was a significant effect of training 
condition, such that transfer scores were lower overall with trials-to-criterion training than fixed training, $F(1,234)=10.97, p=.001, \eta_{p}^{2}=.045$. Although there was a significant effect of training condition, there was no significant interaction between training condition and trial-sequencing, $F(2,234)=.467, p=.628, \eta_{p}^{2}=.004$. Collapsing across both training groups, transfer scores were significantly lower in the intermixed group than the average of blocked and clustered groups, $F(1,234)=14.72, p<.001, \eta_{p}^{2}=.059$. There was no significant difference between the blocked and clustered groups, $F(1,234)=1.49, p=.224, \eta_{p}^{2}=.006$. In this case, there was also a significant association between trial-sequencing group and the proportion of participants exhibiting a positive transfer score, $X(2)=6.89, p=.032$.

Learning scores for trained items from the complete and incomplete discriminations are shown in Figure 11. For the complete discriminations, accuracy was significantly poorer in the intermixed condition than the average of the experimental conditions, $F(1,234)=5.97$, $p=.015, \eta_{p}^{2}=.025$, but there was no significant difference between blocked and clustered conditions, $F(1,234)=1.36, p=.245, \eta_{p}^{2}=.006$. Neither contrast reached significance for the incomplete trained items, highest $F(1,234)=3.63, p=.058, \eta_{p}^{2}<.001$.

\section{Individual differences}

Correlations between transfer score and individual difference measures are shown in Table 9. There was a significant positive correlation between transfer score and CRT score, $r$ $=.135, p=.036$. In this case, this was driven primarily by the blocked group, $r=.262, p=$ .019 , with no significant correlation in the intermixed group, $r=.07, p=.536$, or clustered group, $r=.091, p=.424$. There was also a significant correlation between transfer scores and accuracy on the complete discrimination test items. That is, the better participants retrieved the training contingencies at test, the greater rule transfer, $r=.360, p<.001$. There was no significant correlation between RPM and relational transfer, $r=.114, p=.222$. Correlations 
by group ranged from $r=-.024, p=.883$ in the intermixed group, to $r=.275, p=.090$ in the blocked group.

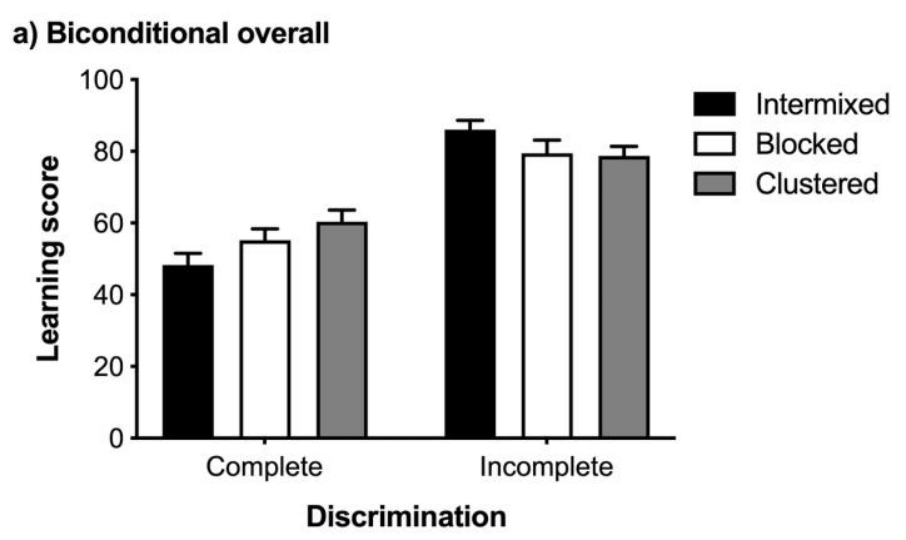

b) Biconditional fixed

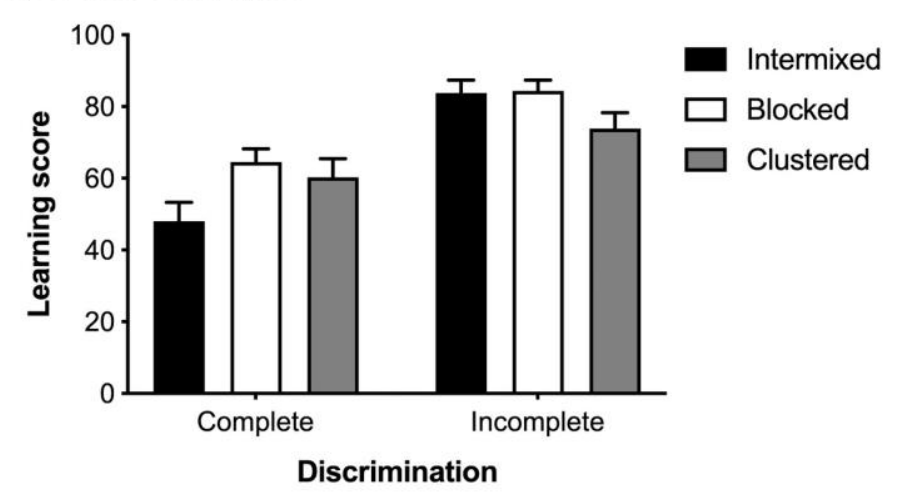

c) Biconditional criterion

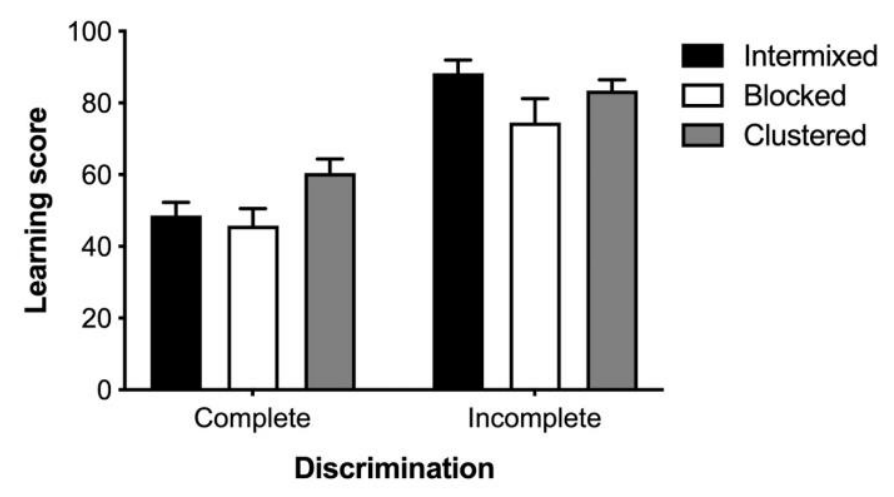

Figure 11. Learning scores at test for the trained items from the complete and incomplete biconditional discriminations a) overall, b) in the fixed training condition, and c) in the trialsto-criterion training criterion. 
Table 9.

Correlation matrix for all biconditional participants

\begin{tabular}{|c|c|c|c|c|c|c|}
\hline & & Transfer & CRT & Ravens & $\begin{array}{c}\text { Test } \\
\text { complete }\end{array}$ & $\begin{array}{c}\text { Test } \\
\text { incomplete }\end{array}$ \\
\hline \multirow[t]{3}{*}{ CRT } & $r$ & $.135 *$ & - & & & \\
\hline & $p$ & .036 & & & & \\
\hline & $n$ & 240 & & & & \\
\hline \multirow[t]{3}{*}{ Ravens } & $r$ & .114 & .503 & - & & \\
\hline & $p$ & .222 & $<.001$ & & & \\
\hline & $n$ & 116 & 116 & & & \\
\hline \multirow[t]{3}{*}{ Test complete } & $r$ & .360 & .205 & .292 & - & \\
\hline & $p$ & $<.001$ & .001 & .001 & & \\
\hline & $n$ & 240 & 240 & 116 & & \\
\hline \multirow[t]{3}{*}{ Test incomplete } & $r$ & .049 & .169 & .155 & .487 & - \\
\hline & $p$ & .448 & .009 & .097 & $<.001$ & \\
\hline & $n$ & 240 & 240 & 116 & 240 & \\
\hline
\end{tabular}

\section{Discussion}

Experiment 3 shows an effect of trial-sequencing on rule transfer for a biconditional discrimination. The effects of these sequences differed from those observed for patterning discriminations in Experiment 2. Overall, the two experimental conditions resulted in improved rule transfer compared to the intermixed condition, which showed stronger featurebased transfer. Unlike the previous experiment, the blocked condition led to the highest rate of rule-transfer for the biconditional discrimination. As in Experiment 2, learning scores for the complete discriminations (but not for the incomplete discriminations) were correlated with rule transfer. Replicating the results from Experiment 2, higher scores on the CRT were associated with greater rule transfer, and there was no significant association between rule transfer and RPM performance.

Even with trial-sequencing manipulations designed to encourage learning of the relations, the biconditional discrimination was still relatively difficult compared to the patterning discrimination. Blocked sequencing improved training performance to a comparable level to intermixed patterning conditions, however learning scores for complete discriminations at test in all three groups were still low compared to the performance of the 
intermixed patterning group in Experiment 2. It is also worth noting, that the effect of learning sequence condition on recall of complete discriminations differed between the two complex discriminations. In Experiment 2, the intermixed condition elicited the highest learning scores and the blocked elicited the lowest, while in Experiment 3 the intermixed condition elicited the lowest learning scores, and learning scores were higher in the blocked and clustered condition.

\section{General Discussion}

This series of experiments examined the influence of training conditions on relational transfer for a relatively simple, and a relatively complex set of relational structures in discrimination learning. We found that the amount of training had little effect on relational transfer in either discrimination, while trial-sequencing had different effects depending on the type of discrimination. We will elaborate on each of these findings in turn.

\section{Training length}

It is perhaps surprising that the amount of training had such a modest effect on participants' tendency to show rule-based transfer. In Experiment 1, a greater than two-fold increase in the number of training trials resulted in an increase in accuracy during training but only negligible (and non-significant) increases in rule transfer. There was also no significant relationship between training accuracy and rule transfer across all participants. The manipulations of trial sequencing used in Experiments 2A and 3A held training length constant. On average, participants completing the trials-to-criterion versions of these experiments observed considerably fewer trials of the complete discriminations than participants completing the fixed-length versions. With this in mind, it is noteworthy that the trials-to-criterion version of the biconditional task (Experiment 3B) produced less rule-based transfer than the fixed-length version (Experiment 3A). This, at least, is consistent with the hypothesis that rule transfer is positively related to the amount of learning that has taken 
place, though the difference could be attributed to other incidental differences between Experiments 3A and 3B (e.g., the time of testing), and a similar difference between Experiments $2 \mathrm{~A}$ and $2 \mathrm{~B}$ is not so evident despite there being a more substantial difference in training length in this experiment (due to patterning being easier to reach criterion). On balance, there may be some effect of the amount of training but the lack of consistent evidence is striking. Although the amount of training appears to have had little impact on the tendency towards rule transfer in this task, it is still possible that those with more efficient associative learning ultimately show more rule transfer, consistent with Shanks and Darby's (1998) hypothesis. Rather than a direct link between training performance and rule-based transfer, we suggest that there may be a more indirect link between training performance and rule-based transfer, in which rule discovery results in both more efficient learning, and greater use of rule transfer. Our results suggest that rule discovery may not be based on overall experience with the stimuli, but in the design of a learning task (and a propensity for cognitive reflection) that supports structural alignment processes.

It is possible that the choice of learning task contributed to this result to some extent. The food allergist task capitalises on familiar stimuli that participants learn about quickly and intuitively, but it also presents the opposites rule in a way that may seem counterintuitive. The type of participant who learns about food-allergic reaction relationships quickly may struggle to accept that food combinations can cause allergic reactions where the constituent foods do not or, perhaps even more counter-intuitively, that combinations of allergenic foods could result in no adverse reaction. There is strong precedence for using the food allergist task to study rule-transfer in this task (e.g. Don et al., 2016; Shanks \& Darby, 1998; Wills et al., 2011). Nevertheless, future research will be needed to ascertain whether our trial-length and trial-sequencing results generalise to other learning tasks in which patterning ruletransfer has been investigated (e.g. Cobos et al., 2017). 


\section{Trial-sequencing}

Experiment 2 and 3 manipulated the sequence in which elements from each discrimination were presented. The effect of these manipulations differed according to the type of discrimination. Blocking all repetitions of the elements from one discrimination within a single block led to greater training accuracy in both discriminations. While this condition was detrimental for rule transfer for a more simple relational rule (patterning), it was beneficial for a more difficult relational rule (biconditional). Clustering trials, such that the discrimination sets were intermixed but all elements of a discrimination set were experienced in a cluster of consecutive trials, led to better rule transfer for both relatively simple and relatively complex relational structures.

Why would blocking trials be effective? Blocking trials of the same discrimination together may be effective as it enables comparison of relevant elements within each instance of the relation. This kind of comparison within instances of the discrimination may be more important for a more complex set of relations. The biconditional task involves a large number of cues that appear in multiple compounds. In this case, blocking together compounds from one instance of the rule may serve to highlight the relationship between the specific combinations of cues and outcomes that form the basis of the relational rule.

For the simpler relational structure (patterning), it appears that the consecutive repetition of the same elements in the blocked condition paradoxically leads to inflexibility in transferring the relational rule. The blocked patterning condition was far and away the easiest and should be the condition in which the relational structure is most obvious. Yet this condition showed the least flexibility in extending the rule to novel stimuli. We hypothesise that this pattern is a consequence of the learning task being easy and repetitive enough to perform accurately without any deep encoding (that is, without rule discovery and potentially without even committing cue-outcome relationships to long-term memory). Consistent with 
this, memory for the complete discriminations at test was quite poor despite very high accuracy during training. This pattern directly contrasts with the effect of blocking the biconditional discriminations where the relational structure is harder to discover, and thus consecutively repeating elements in the blocked condition instead serves to help rule transfer.

Why would clustering trials be effective? Clustering trials enables both the comparison of relevant elements within each instance of the relational structure that defines the complex discrimination, but also comparison across instances of these relations. These comparisons may increase the likelihood that participants abstract and apply the relational rule. This is the sequence that structural alignment theories (e.g. Goldwater \& Gentner, 2015) predict should maximise learning and transfer. The variability of instances of the relation over the course of training also prevents the kind of surface-level solution that the blocked condition affords when the rule is more simple, but is still advantageous (compared to standard random intermixing) when the rule is complex. In addition, clustering trials in this way may still afford strong memory retention in a similar way to intermixing trials because the instances of encoding cue-outcome relationships are still spaced out across training.

\section{Desirable difficulties and the connection between learning and performance}

One potential explanation for the differences in effects of trial sequences between discriminations is desirable difficulty (Bjork, 1994). Difficulty during learning can increase long-term retention and transfer, but only when the difficulty does not exceed cognitive capacities. This work points to how, for example, testing previously studied learning materials is both more difficult than re-studying those same materials, and leads to better long term learning outcomes (see Bjork, Dunlovksy \& Kornell, 2013 for review). However, work on cognitive load theory argues that when the learning materials themselves are of sufficient complexity, the benefits of difficult learning tasks, such as tests of problem solving may disappear (e.g., Leahy, Hanham, \& Sweller, 2015). Likewise in the current paper, when the 
relation is simpler, like the patterning rule, more difficult training sequences might lead to deeper processing and subsequently better transfer of the relational structure. In contrast, blocking trials during training may be too simple, and therefore result in shallower processing. When the relational rule is already complex, like the biconditional rule, more difficult training sequences may no longer be effective, and these relational rules may benefit from conditions that improve performance in training.

\section{Learning and relational transfer}

Considering the pattern of results across all the experiments, the relationship between performance during training and the subsequent measures of memory and transfer is complex. In Experiment 2, although accuracy during training was highest for the blocked group, learning scores for the trained elements from the complete discriminations was poorest in this group, which might indicate poor processing of the contingencies during training. This suggests that high accuracy during training can potentially be achieved without any "true" learning. In comparison, in Experiment 3, blocking trials in the biconditional discrimination led to comparatively better rating accuracy for complete discriminations at test, and likewise elicited higher rates of rule transfer than in Experiment 2.

An alternative explanation for the performance in the patterning blocked group is that, during training, only a few trial types were presented at a time, leading to good performance, but at test, many trial types had not been seen for a long time, leading to poor recall. An analysis of recency effects (see Supplemental Material for full analysis) showed that learning scores for the discriminations learned more recently were in fact lower than those that were learned earlier in training, which rules out an account based on recency. This was not the case for the biconditional condition, where recall at test was relatively good, and learning scores were indeed better for more recently learned discriminations. These results instead support our hypothesis that in the case of patterning, once participants realise that they can respond 
accurately on the basis of the most superficial information (e.g., for a given block, one cue means the outcome will occur, two cues means it won't), they are able to complete training with high performance with little encoding of the contingencies, or deeper processing of the relational rule.

In contrast to the blocked group, learning scores for complete discriminations in the intermixed group were relatively high for both experiments, which is consistent with previous findings that intermixing trials is beneficial for memory. However, the intermixed condition never elicited the highest levels of rule transfer. Interestingly, the clustered group showed greater rule transfer than the intermixed group despite having comparable learning scores for the complete discriminations. This complete pattern suggests that while learning the associations might be a necessary condition for rule learning, it is not sufficient for rule learning and transfer.

There is also a methodological implication here. In all supervised learning experiments, experimenters either set a fixed amount of training for all subjects, or instead set a criterion level of accuracy for subjects to achieve, regardless of how long it takes to achieve it. A relatively arbitrary learning criterion is often used to ensure that all participants enter the test phase on equal footing, equating for different learning rates. However, the current work suggests that learning beyond reaching criterion was helpful for rule transfer when the rule was more complex. This suggests that whenever there are multiple available strategies to achieve accurate learning, the use of learning criteria may not be the optimal method.

\section{What causes relational transfer?}

Given that one can achieve high accuracy during training simply by memorizing cueoutcome associations, it may not be the case that all participants would eventually show rule transfer, even if given greatly extended training. Instead, some participants may require conditions that highlight the relational structure more strongly if they are to learn and 
generalise on the basis of relational rules. Looking across Experiments 2 and 3, clustering appears to be the ideal training method, as it has enough trial to trial variety to improve accuracy for individual associations, has enough consistency to highlight the relational structure to improve rule transfer ${ }^{3}$, and seems to not rely on any predisposition for cognitive reflection, as the CRT showed no correlation with transfer in the clustered condition for either discrimination.

On the other hand, the CRT did show correlations with rule transfer in the intermixed condition of Experiment 2 and blocked condition of Experiment 3, perhaps suggesting that under suboptimal learning conditions, a propensity to engage in reflective thinking is supportive of rule transfer. It is worth noting that CRT was also associated with learning of the complete discriminations, and learning for the complete discriminations was associated with rule transfer. If we examine the association between CRT and rule transfer while controlling for complete discrimination learning, the relationship is no longer significant in either patterning or biconditional discriminations (see Supplementary Material). However, it is likely that cognitive reflection aids rule discovery, which would be a common cause affecting both learning efficiency (as measured by complete learning scores), and rule transfer. In this case, it would not be surprising that CRT does not independently predict rule transfer over and above learning scores.

We note that CRT shows a distinct pattern from the relationship with RPM, which shows a greater relation to overall learning than to rule transfer specifically. For example, RPM correlated with learning scores for the complete trained items in Experiment 2, and learning scores for complete and incomplete trained items in Experiment 3, however there were no significant correlations between RPM and rule transfer in either experiment. This is

\footnotetext{
${ }^{3}$ The clustering condition lead to greater relational transfer than intermixed training for both discriminations (see Supplementary Material).
} 
quite consistent with Goldwater et al. (2018)'s work on categorization wherein learners either categorised stimuli by their intrinsic features, or relations among these features. RPM scores predicted overall learning but did not predict whether an individual formed feature-based or relational categories. On the other hand, measures of learning strategy did predict the kind of category that was formed.

There are two interesting connections between Goldwater et al. (2018) and the current study. First, each suggests that whether any individual focuses on relations between items or stimulus features, or the items or features themselves is more an issue of cognitive propensity, strategy, or style, rather than capacity - RPM predicts overall learning in each, but other measures predict either a relational or featural strategy. Second is that both papers identified conditions that dispelled the correlation between cognitive strategy and relational transfer (the clustered condition here, and inference-learning tasks there) by supporting relational transfer regardless of a predisposition to learn relations. As mentioned above, inference-learning tasks present partial exemplars and the goal is to predict the missing elements. To do so, one needs to focus on the relations among the elements. This is a distinct but similarly effective way to support relational learning as the clustering condition in the current work.

\section{Associative learning and memory for complete discriminations}

Associative learning predicts feature based transfer because of the way associations with learned stimulus elements are assumed to combine to generate a prediction when faced with a novel combination of features. A corollary of their operation is that discriminations that are not linearly solvable (including patterning and biconditional discriminations) tend to be more difficult for associative learning networks to master than linearly solvable discriminations. Although even very simple networks do eventually learn patterning and biconditional discriminations (Livesey, Thorwart \& Harris, 2011), they take longer to do so 
and prediction accuracy is thus poorer. The reason for this is because in nonlinear problems, feature-based generalization works strongly against the correct prediction. Take negative patterning for instance, where two cues, A and B, are each paired with the outcome when they occur as individual cues. When the network is given the compound $\mathrm{AB}$, most associative algorithms assume summation of the associative strengths of the individual components $\mathrm{A}$ and $\mathrm{B}$. While this summation may only be partial depending on the particular representational assumptions of the model (see Thorwart, Uengoer, Livesey \& Harris, 2017), it often leads to a particularly strong prediction that the outcome will occur in the presence of $\mathrm{AB}$ when in fact no outcome occurs on these trials. This incorrect prediction needs to be overcome with additional learning, all while retaining learned anticipation of the outcome when A and B are presented separately.

With this in mind, it should be noted that participants across the experiments generally performed better on the incomplete discriminations, which are linearly solvable, than the complete discriminations, which are not (see supplementary materials for further analysis that demonstrates this). This is not a definitive comparison since there are numerous differences between the learning conditions of the complete and incomplete sets, most notably the time of learning (incomplete sets were learned after the complete sets). But it is at least consistent with the idea that associative learning is the foundation on which featurebased learning, and later, extrapolation of relations, is based.

One of the potential benefits of applying a relational rule while solving complex discriminations is that it may provide a means of overcoming the interference caused by feature-based generalization when solving discriminations that do not have a linear solution. If this is the case then it means that rule discovery should enhance rule transfer scores, but potentially also enhance learning scores for the complete discriminations when they are presented on test. We thus expect rule transfer and performance on these complex 
discriminations to be correlated, even if the causal link between them is indirect (that is, there's a common cause for both rule transfer and high performance on the originally learned discriminations).

\section{Towards a model integrating associative and relational learning}

Structural alignment predicts clustering as the sequence to maximise relational rule discovery, and in a broad sense that prediction was borne out. However, structural alignment in the abstract does not make any particular predictions about how sequences would interact with the complexity of the relational rule, which elicited large effects on learning and transfer. This is not for a lack of formal specification, as this process is at the heart of many computational models (e.g., Falkenhainer et al., 1989; Hummel \& Holyoak, 2003). On the other hand, associative learning models are designed to capture these other variables (such as how learning accumulates across trials), but cannot capture how relational rules are discovered and transferred.

The other common class of abstract relational learning models are hierarchical Bayesian models (e.g., Kemp, Tenenbaum, Niyogi \& Griffiths, 2010). One advantage to this approach is that they are designed to account for how people abstract and transfer relational rules from causal learning experiments, and have been developed to integrate both associative and reasoning processes (e.g., Gershman, 2015). Models such as that proposed by Kemp et al. (2010) posit that learning about individual causal relationships, and classes of causal relationships that generalise across stimuli, is a unified process. This provides an elegant formal solution. However, it appears that these models do not make predictions that would dissociate relational from associative learning based on trial quantity or trial sequence (again, as abstraction and instance learning is a unified process), and so we do not believe that these models could account for the current pattern of results without further modifications. 
Going forward, models should be developed to integrate associative and relational learning mechanisms. In the domain of reinforcement learning, there has been work to integrate associative learning processes (e.g., "model-free learning”) with more workingmemory dependent structured learning strategies (e.g., "model-based learning”). One consideration of these models is whether there needs to be some kind of meta-control process that decides which process to engage (e.g., Kool, Gershman, \& Kushman, 2017). We suggest a similar process would be needed here, that would for example, be sensitive to difficulty during training. This paper has established clear empirical benchmarks for any such account to capture, including learning structure conditions (such as clustering) that may trigger the engagement of structure-learning processes, and identifying which individuals (as measured by tasks such as the CRT) are more likely to engage these processes regardless.

\section{Acknowledgements}

This research was funded by Australian Research Council Grant DP150104267 awarded to Micah B. Goldwater and Evan J. Livesey. Data are available on the Open Science Framework https://osf.io/m7628/ 


\section{References}

Baetu, I., Burns, N. R., Yu, E., \& Baker, A. G. (2018). Fluid Abilities and Rule Learning: Patterning and Biconditional Discriminations. Journal of Intelligence, 6, 7; doi:10.3390/jintelligence6010007.

Bjork, R. A. (1994). Memory and metamemory considerations in the training of human beings. In J. Metcalfe \& A. P. Shimamura (Eds.), Metacognition: Knowing about knowing (pp. 185-205). Cambridge, MA, US: The MIT Press.

Bjork, R. A., Dunlosky, J., \& Kornell, N. (2013). Self-regulated learning: Beliefs, techniques, and illusions. Annual review of psychology, 64, 417-444.

Carvalho, P. F., \& Goldstone, R. L. (2014). Putting category learning in order: Category structure and temporal arrangement affect the benefit of interleaved over blocked study. Memory \& cognition, 42, 481-495.

Carvalho, P. F., \& Goldstone, R. L. (2015). The benefits of interleaved and blocked study: Different tasks benefit from different schedules of study. Psychonomic bulletin \& review, 22, 281-288.

Catrambone, R., \& Holyoak, K. J. (1989). Overcoming contextual limitations on problemsolving transfer. Journal of Experimental Psychology: Learning, Memory, and Cognition, 15, 1147.

Cobos, P. L., Gutiérrez-Cobo, M. J., Morís, J., \& Luque, D. (2017). Dependent measure and time constraints modulate the competition between conflicting feature-based and rulebased generalization processes. Journal of Experimental Psychology: Learning, Memory, and Cognition, 43, 515-. 
Don, H., Goldwater, M., \& Livesey, E. J. (2019, September 28). Relational rule discovery in complex discrimination learning. Retrieved from osf.io/m7628

Don, H. J., Goldwater, M. B., Otto, R. A., \& Livesey, E. J. (2015). Connecting ruleabstraction and model-based choice across disparate learning tasks. In D. Noelle, R. Dale, A. Warlaumont, J. Yoshimi, T. Matlock, C. Jennings, \& P. Maglio (Eds.), Proceedings of the 37th Annual Conference of the Cognitive Science Society (pp. 590-595). Pasadena, California: Cognitive Science Society.

Don, H. J., Goldwater, M. B., Otto, R., Livesey, E. J. (2016). Rule abstraction, model-based choice and cognitive reflection. Psychonomic Bulletin \& Review, 23, 1615-1623.

Doumas, L. A., Hummel, J. E., \& Sandhofer, C. M. (2008). A theory of the discovery and predication of relational concepts. Psychological review, 115, 1.

Ebbinghaus, H. (1885). Memory: A contribution to experimental psychology, trans. HA Ruger \& CE Bussenius. Teachers College [rWvH].

Falkenhainer, B., Forbus, K. D., \& Gentner, D. (1989). The structure-mapping engine: Algorithm and examples. Artificial intelligence, 41, 1-63.

Frederick, S. (2005). Cognitive reflection and decision making. Journal of Economic perspectives, 19, 25-42.

Gentner, D. (2003). Why we're so smart. In D. Gentner \& S. Goldin-Meadow (Eds.), Language in mind: Advances in the study of language and thought. Cambridge, MA: MIT Press. 
Gentner, D., \& Kurtz, K. J. (2005). Relational categories. In W.-K. Ahn (Ed.), Categorization inside and outside the lab (pp. 151-175). Washington, DC: American Psychological Association.

Gershman, S. J. (2015). A unifying probabilistic view of associative learning. PLoS computational biology, 11(11), e1004567.

Gick, M. L., \& Holyoak, K. J. (1983). Schema induction and analogical transfer. Cognitive psychology, 15(1), 1-38.

Goldwater, M. B., Don, H. J., Krusche, M., \& Livesey, E. J. (2018). Relational Discovery in Category Learning, Journal of Experimental Psychology: General, 147, 1-35.

Goldwater, M. B., \& Gentner, D. (2015). On the acquisition of abstract knowledge: Structural alignment and explication in learning causal system categories. Cognition, 137, 137-153.

Goldwater, M. B., \& Schalk, L. (2016). Relational categories as a bridge between cognitive and educational research. Psychological Bulletin, 142, 729-757.

Gray, M. E., \& Holyoak, K. J. (2019). Individual differences in relational reasoning. Memory \& Cognition, 1-15.

Leahy, W., Hanham, J., \& Sweller, J. (2015). High element interactivity information during problem solving may lead to failure to obtain the testing effect. Educational Psychology Review, 27, 291-304.

Halford, G. S., Wilson, W. H., \& Phillips, S. (2010). Relational knowledge: The foundation of higher cognition. Trends in cognitive sciences, 14, 497-505. 
Harris, J. A., Livesey, E. J., Ghareai, S., \& Westbrook, R. F. (2008). Negative patterning is easier than a biconditional discrimination. Journal of Experimental Psychology: Animal Behavior Processes, 34, 494-500.

Harris, J. A. \& Livesey, E. J. (2008). Comparing Patterning and Biconditional Discriminations in Humans. Journal of Experimental Psychology: Animal Behavior Processes, 34, 144-154.

Holyoak, K. J., \& Thagard, P. (1989). Analogical mapping by constraint satisfaction. Cognitive Science, 13, 295-355.

Hummel, J. E., \& Holyoak, K. J. (2003). A symbolic-connectionist theory of relational inference and generalization. Psychological review, 110, 220.

Kemp, C., Goodman, N. D., \& Tenenbaum, J. B. (2010). Learning to learn causal models. Cognitive Science, 34, 1185-1243.

Kleiner, M., Brainard, D., \& Pelli, D. (2007). What's new in psychtoolbox-3. Perception, 36, ECVP Abstract Supplement.

Kokinov, B., \& Petrov, A. (2001). Integrating memory and reasoning in analogy-making: The AMBR model. The analogical mind: Perspectives from cognitive science, 59-124.

Kool, W., Gershman, S. J., \& Cushman, F. A. (2017). Cost-benefit arbitration between multiple reinforcement-learning systems. Psychological science, 28, 1321-1333.

Little, J. L., \& McDaniel, M. A. (2015). Individual differences in category learning: Memorization versus rule abstraction. Memory \& Cognition, 43, 283-297. 
Livesey, E. J., Don, H. J., Uengoer, M., \& Thorwart, A. (2019). Transfer of associability and relational structure in human associative learning. Journal of Experimental Psychology: Animal Learning \& Cognition, DOI: 10.1037/xan0000197

Livesey, E. J., Thorwart, A., \& Harris, J. A. (2011). Comparing positive and negative patterning in human learning. Quarterly Journal of Experimental Psychology, 64, 23162333.

Livesey, E. J., Thorwart, A., De Fina, N. L., \& Harris, J. A. (2011). Comparing learned predictiveness effects within and across compound discriminations. Journal of Experimental Psychology: Animal Behavior Processes, 37, 446-465.

Maes, E., De Filippo, G., Inkster, A. B., Lea, S. E., De Houwer, J., D’Hooge, R., ... \& Wills, A. J. (2015). Feature-versus rule-based generalization in rats, pigeons and humans. Animal Cognition, 18, 1267-1284.

Maes, E., Vanderoost, E., D'Hooge, R., De Houwer, J., \& Beckers, T. (2017). Individual difference factors in the learning and transfer of patterning discriminations. Frontiers in psychology, 8:1262.

McDaniel, M. A., Cahill, M. J., Robbins, M., \& Wiener, C. (2014). Individual differences in learning and transfer: Stable tendencies for learning exemplars versus abstracting rules. Journal of Experimental Psychology: General, 143, 668.

Milton, F., Bealing, P., Carpenter, K. L., Bennattayallah, A., \& Wills, A. J. (2017). The neural correlates of similarity-and rule-based generalization. Journal of Cognitive Neuroscience, 29, 150-166. 
Penn, D. C., Holyoak, K. J., \& Povinelli, D. J. (2008). Darwin's mistake: Explaining the discontinuity between human and nonhuman minds. Behavioral and Brain Sciences, 31, 109-130.

Rohrer, D., \& Pashler, H. (2010). Recent research on human learning challenges conventional instructional strategies. Educational Researcher, 39, 406 - 412

Saavedra, M. A. (1975). Pavlovian compound conditioning in the rabbit. Learning and Motivation, 6, 314-326.

Shanks, D. R., \& Darby, R. J. (1998). Feature- and rule-based generalization in human associative learning. Journal of Experimental Psychology: Animal Behavior Processes, 24, 405-415.

Thorwart, A., Livesey, E. J. \& Harris, J. A. (2012). Normalisation between stimulus elements in a model of Pavlovian conditioning: Showjumping on an elemental horse. Learning \& Behavior, 40, 334-346.

Thorwart, A., Uengoer, M., Livesey, E. J. \& Harris, J. A. (2017). Summation effects in human learning: evidence from patterning discriminations in goal-tracking. Quarterly Journal of Experimental Psychology, 70, 1366-1379.

Tohill, J. M., \& Holyoak, K. J. (2000). The impact of anxiety on analogical reasoning. Thinking \& Reasoning, 6, 27-40.

Toplak, M. E., West, R. F., \& Stanovich, K. E. (2011). The Cognitive Reflection Test as a predictor of performance on heuristics-and-biases tasks. Memory \& cognition, 39, 1275-.

Wills, A. J., Barrasin, T. J., \& McLaren, I. P. L. (2011). Working memory capacity and generalization in predictive learning. In L. Carlson, C. Hölscher, \& T. Shipley (Eds.), 
Proceedings of the 33rd annual conference of the Cognitive Science Society (pp. 32053210). Austin, TX: Cognitive Science Society.

Wills, A. J., Graham, S., Koh, Z., McLaren, I. P. L., \& Rolland, M. D. (2011). Effects of concurrent load on feature- and rule-based generalization in human contingency learning. Journal of Experimental Psychology: Animal Behavior Processes, 37, 308-316. 